\title{
Direct numerical simulation and subgrid analysis of a transitional droplet laden mixing layer
}

Richard S. Miller, and Josette Bellan

Citation: Physics of Fluids 12, 650 (2000); doi: 10.1063/1.870271

View online: http://dx.doi.org/10.1063/1.870271

View Table of Contents: http://aip.scitation.org/toc/phf/12/3

Published by the American Institute of Physics

\section{Articles you may be interested in}

A dynamic subgrid-scale eddy viscosity model

Physics of Fluids A: Fluid Dynamics 3, 1760 (1998); 10.1063/1.857955

A dynamic subgrid-scale model for compressible turbulence and scalar transport

Physics of Fluids A: Fluid Dynamics 3, 2746 (1998); 10.1063/1.858164

A large-eddy simulation scheme for turbulent reacting flows

Physics of Fluids A: Fluid Dynamics 5, 1282 (1998); 10.1063/1.858617

Turbulence-flame interactions in DNS of a laboratory high Karlovitz premixed turbulent jet flame

Physics of Fluids 28, 095107 (2016); 10.1063/1.4962501

Direct numerical simulation of a transitional temporal mixing layer laden with multicomponent-fuel evaporating drops using continuous thermodynamics

Physics of Fluids 16, 1884 (2004); 10.1063/1.1688327

A priori subgrid analysis of temporal mixing layers with evaporating droplets

Physics of Fluids 12, 1573 (2000); 10.1063/1.870405

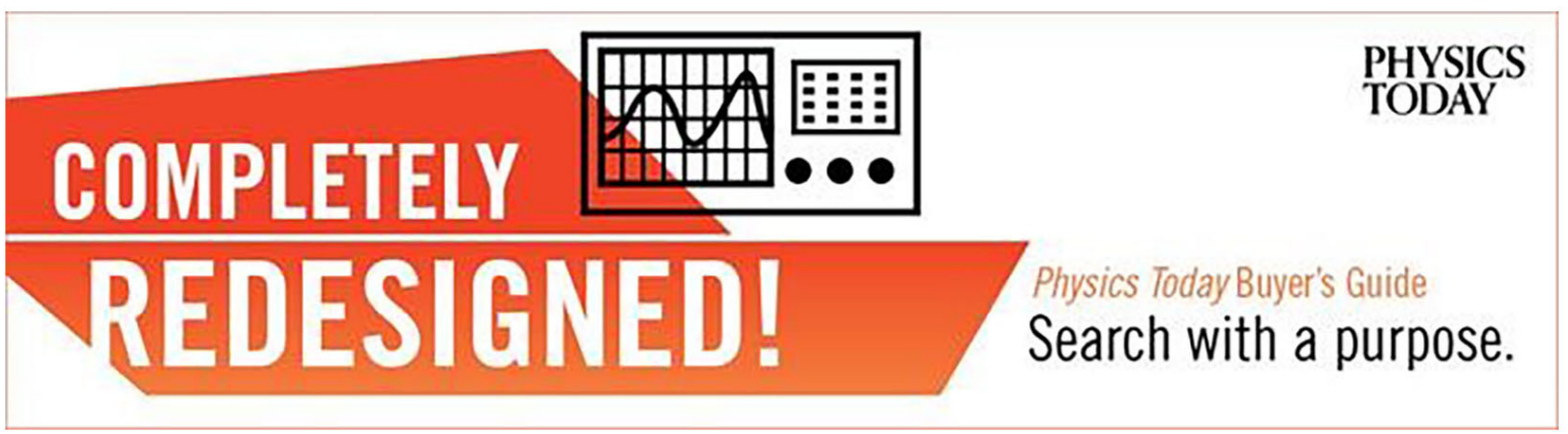




\title{
Direct numerical simulation and subgrid analysis of a transitional droplet laden mixing layer
}

\author{
Richard S. Miller ${ }^{\mathrm{a})}$ and Josette Bellan \\ Jet Propulsion Laboratory, California Institute of Technology, Pasadena, California 91109-8099
}

(Received 13 February 1999; accepted 1 December 1999)

\begin{abstract}
Direct numerical simulations of a temporally developing, droplet laden mixing layer undergoing transition to mixing turbulence are conducted. The formulation includes complete two-way couplings of mass, momentum, and energy. As many as $18 \times 10^{6}$ grid points are used to discretize the Eulerian gas phase equations and up to $5.7 \times 10^{6}$ initially polydisperse evaporating droplets are tracked in the Lagrangian reference frame. The complete transition to mixing turbulence is captured for several of the higher Reynolds number simulations and it is observed that increasing the droplet mass loading ratio results in a more "natural" turbulence characterized by increased rotational energy and less influence of the initial forcing perturbations. An increased mass loading also results in increased droplet organization within the layer. An a priori subgrid analysis is then conducted which shows that neglecting subgrid velocity fluctuations in the context of large eddy simulations may result in significant errors in predicting the droplet drag force for Stokes numbers St $\sim 1$ (with the flow time scale based on the mean velocity difference and initial vorticity thickness). Similar possible errors of lesser magnitude are also observed for the droplet heat flux and evaporation rate when thermodynamic subgrid fluctuations are neglected. An extension of the eddy interaction model commonly used in Reynolds-averaged simulations is then proposed in order to account for the missing subgrid information. Probability density functions (PDFs) of the subgrid fluctuations calculated across homogeneous planes are shown to be highly intermittent, particularly near the laminar-turbulent boundaries of the mixing layer. However, the actual subgrid PDFs calculated locally are much less intermittent and may be adequately modeled by the Gaussian distribution throughout the majority of the mixing layer. A scale similarity model is then employed to predict both the velocity and thermodynamic subgrid variances. The similarity model is well correlated with the actual subgrid variances and shows good agreement in predicting the local fluctuation intensities when a filter width-dependent model constant is used. The subgrid fluctuation variances acting on the droplets are then shown to be well modeled if the Eulerian subgrid variance model is interpolated to the droplet locations. [S1070-6631(00)02303-5]
\end{abstract}

\section{INTRODUCTION}

Despite many recent advances in the direct numerical simulation (DNS) of turbulent two-phase flows, ${ }^{1}$ the DNS of "two-way coupled" flows in which the dispersed phase mass loadings are sufficiently large to modulate the turbulence are sparse. For these flows, large numbers $\left(\sim 10^{5}\right.$ $\rightarrow \sim 10^{6}$ ) of individual dispersed particles are tracked in the Lagrangian reference frame and the calculation of the coupling source terms appearing in the continuous phase transport equations results in a substantial computational cost increase relative to one-way coupled flows. Flows considered in this manner are generally restricted to those characterized by a dispersed phase composed of discrete spherical elements having small volume ratios $\left(\leqslant 10^{-3}\right)$, but moderate mass loading ratios $\left(\sim 10^{-1} \rightarrow \sim 10^{0}\right)$ due to large ratios of the dispersed to carrier phase densities. Practical applications

\footnotetext{
a) Author to whom correspondence should be addressed. Present address: Department of Mechanical Engineering, Clemson University, Clemson, SC 29634-0921; electronic mail: richard.miller@ces.clemson.edu
}

for flows requiring two-way coupled descriptions are widespread, including both solid particles and liquid droplet flows; e.g., pulverized coal combustion, spray painting, spray combustion, atomization, weather prediction, etc.

Two-way coupled DNS of incompressible box turbulence laden with solid particles have been performed in several past studies ${ }^{2-6}$ by tracking as many as $10^{6}$ individual particles. For these solid particle flows the only coupling term is the particle drag force that appears in the carrier phase momentum equation. In these studies it is observed that the turbulence is selectively modulated by the particles due to the preferential concentration mechanism through which particles tend to concentrate in high strain (lowvorticity) regions of the flow (e.g., Ref. 1). In the absence of gravity, the total turbulence energy is diminished by the particle drag; however, a relative increase in the high wave number carrier gas energy spectrum is found. ${ }^{3,6}$ Gravitational acceleration of the particles imparts anisotropy to the turbulence by adding energy to the gas phase momentum component parallel to the acceleration. This energy is then transferred to the other velocity components through the 
pressure-strain correlation. Elghobashi and Truesdell ${ }^{3}$ postulate that this process results in a reverse cascade of energy that can reduce turbulence decay rates relative to either unladen or zero gravity flows.

Evaporating droplet laden flows incur substantially heavier computational costs than solid particle flows due to the following: (1) additional transport equations for the droplet mass and temperature, (2) additional mass and energy coupling terms, and (3) the need to treat the gas phase equations using a compressible (or variable density) formulation. Mashayek $^{7,8}$ conducted DNS of both isotropic and homogeneous shear box turbulence using an energy coupling formulation that is strictly valid only for constant and equal liquid and vapor heat capacities. Mashayek's studies tracked as many as $5.5 \times 10^{5}$ evaporating droplets obeying a "mass analogy" evaporation law" with complete mass, momentum, and energy coupling, and solving the compressible NavierStokes equations for the gas phase carrier flow. Recently Miller and Bellan ${ }^{10}$ (hereinafter referred to as MBI) extended two-way coupled DNS to nonhomogeneous flows with simulations of a (pre-transitional) planar mixing layer having one stream laden with as many as $7.3 \times 10^{5}$ evaporating droplets. Their formulation extends that of Mashayek and is valid for varying liquid and vapor heat capacities. Furthermore, they employ the nonequilibrium Langmuir-Knudsen law to describe the droplet evaporation that has been shown to be valid over a broader range of conditions than either the mass analogy or standard equilibrium rapid mixing models. ${ }^{9}$ The MBI simulations showed that at low gas temperatures the droplet laden stream rapidly saturates due to the buildup of evaporated vapor and cooling of the gas temperature resulting from latent heat effects. After this saturation occurs, the droplets in the free stream essentially cease vaporizing; however, droplets near the mixing region become engulfed into the layer where they come into contact with higher temperature carrier gas and continue to evaporate. This results in a large disparity between the droplet size distributions found in the interior and laden stream portions of mixing layers. Miller and Bellan ${ }^{11}$ have also used the same simulation database to address assumed probability density function (PDF) modeling of nonpremixed combustion in Reynolds-averaged Navier-Stokes (RANS) simulations. They derived the necessary mixture fraction moment transport equations, analyzed their budgets, and observed that the assumed $\beta$ PDF poorly represents the simulated scalar fields.

A different approach from DNS is large eddy simulation (LES) in which the governing equations rather than being resolved down to the Kolmogorov scale are instead filtered locally in either physical or wave number space; therefore, the effects of the small scales of the flow field must be modeled. ${ }^{12,13}$ Although generally employed for single phase flow predictions, LES is also used to study particle dispersion. ${ }^{14-19}$ In reality, the particles are convected by the superimposed effects of both the resolved (filtered) velocity field and the unresolved subgrid velocity fluctuations. However, in the cited references the subgrid effects were always neglected under the assumption that the particle inertia is sufficiently large; thus the particles should not significantly interact with the small-scale flow motions. This assumption has never been tested against either DNS or experimental data, and the actual influence of subgrid fluctuations may have a significant effect on dispersion, even for particles with Stokes numbers $\mathrm{St} \sim 1$ depending on the flow Reynolds number and the relative filter width. The situation becomes more complex for evaporating droplets due to the additional effects of subgrid thermodynamic fluctuations that affect both the droplet heating and the vaporization rates. One exception to the above references is Pannala and Menon, ${ }^{20}$ who perform LES of droplet laden turbulence using a modified eddy interaction model (EIM) to account for subgrid velocity (but not thermodynamic) fluctuations. The subgrid variance is provided by an additional transport equation for the subgrid kinetic energy; however, the EIM was simply extended without further comment, and no a priori testing was mentioned. Also, using the subgrid kinetic energy equation for the fluctuation variances is not directly applicable for flows in which the subgrid velocity may be anisotropic.

The objectives of the present paper are to (1) extend the simulations of MBI to sufficiently high Reynolds numbers to capture the transition to mixing turbulence for multiphase flows, where both evaporation and complete two-way coupling are important; (2) use the simulated database to perform an a priori investigation of the role of subgrid fluctuations on the transport, heating, and vaporization of droplets in the context of LES; and (3) to propose a model for these subgrid effects. A discussion of methods for treating the phase coupling terms in an actual LES is deferred to a further publication. The mathematical formulation and numerical approach are described in Sec II. Results are presented in Sec. III; this section includes a detailed description of the transition to turbulence and the corresponding effects of the flow Reynolds number and droplet mass loading ratio. Subgrid fluctuations are then investigated based on filtering the resolved DNS fields using both spherical and cubic spatial filter kernels. An extension of the EIM, which is tested $a$ priori, is then proposed to model both velocity and thermodynamic subgrid fields for LES, which is based on a scale similarity model to predict subgrid variances. Section IV contains conclusions and further discussions.

\section{FORMULATION AND APPROACH}

The governing equations describe the Lagrangian transport of discrete evaporating droplets through a continuous and calorically perfect carrier gas flow. These equations were described in detail in $\mathrm{MBI}^{10}$ and will therefore only be summarized here. The only difference between the equations described below and those used in MBI is that in the present paper we choose to neglect thermodynamic nonequilibrium effects that are known to be insignificant for the droplet sizes $(\sim 100 \mu \mathrm{m})$ and relatively low gas temperatures of interest in this study. ${ }^{9}$ In this case the model reduces to the classical rapid mixing model; i.e., the " $D^{2}$ law" combined with a transient droplet temperature equation. Throughout the formulation, the subscripts $C, V$, and $L$ will be used to distinguish quantities specific to the carrier gas, the evaporated vapor, and the liquid, respectively; whereas the subscript $G$ refers to the carrier plus vapor gas phase mixture. The com- 
pressible form of the governing equations for the gas phase (carrier plus vapor mixture) include mass, momentum, and energy exchange between the gas and the dispersed evaporating liquid phase:

$$
\begin{aligned}
& \frac{\partial \rho}{\partial t}+\frac{\partial}{\partial x_{j}}\left[\rho u_{j}\right]=S_{\mathrm{I}}, \\
& \frac{\partial}{\partial t}\left(\rho u_{i}\right)+\frac{\partial}{\partial x_{j}}\left[\rho u_{i} u_{j}+P \delta_{i j}-\tau_{i j}\right]=S_{\mathrm{II}, i}, \\
& \frac{\partial}{\partial t}\left(\rho e_{t}\right)+\frac{\partial}{\partial x_{j}}\left[\left(\rho e_{t}+P\right) u_{j}-\lambda \frac{\partial T}{\partial x_{j}}-u_{i} \tau_{i j}\right]=S_{\mathrm{III}}, \\
& \frac{\partial}{\partial t}\left(\rho Y_{V}\right)+\frac{\partial}{\partial x_{j}}\left[\rho Y_{V} u_{j}-\rho \Gamma \frac{\partial Y_{V}}{\partial x_{j}}\right]=S_{\mathrm{I}}, \\
& P=\rho\left[Y_{V} R_{V}+\left(1-Y_{V}\right) R_{C}\right] T,
\end{aligned}
$$

where $\rho$ is the gas phase density, $u_{i}$ is the gas phase velocity, $e_{t}=e+u_{i} u_{i} / 2$ is the total gas energy, i.e., kinetic energy plus internal energy $\left[e=\left(1-Y_{V}\right) C_{\mathrm{v}, C} T+Y_{V}\left(C_{\mathrm{v}, V} T+h_{V}^{0}\right)\right.$ with vapor reference enthalpy $\left.h_{V}^{0}\right], P$ is the thermodynamic pressure, $Y_{V}$ is the mass fraction of the evaporated vapor, and $R_{C}$ and $R_{V}$ are the carrier gas (subscript $C$ ) and vapor (subscript $V$ ) gas constants (i.e., $R=\Re / W$ with universal gas constant $\mathfrak{R}$ and molecular weight $W$ ). Furthermore, $\tau_{i j}$ is the Newtonian viscous stress tensor and $\mu, \lambda$, and $\Gamma$ are the gas phase viscosity, thermal conductivity, and Fickian diffusion coefficient, respectively (hereinafter assumed to be constants). The right-hand side (rhs) terms $S_{\mathrm{I}}, S_{\mathrm{II}, i}$, and $S_{\mathrm{III}}$ describing the phase couplings of mass, momentum, and energy, respectively, are defined below.

The modeled Lagrangian equations describing the transient position $\left(X_{i}\right)$, velocity $\left(\mathrm{V}_{i}\right)$, temperature $\left(T_{d}\right)$, and mass $\left(m_{d}\right)$ of a single droplet are

$$
\begin{aligned}
& \frac{d X_{i}}{d t}=\mathrm{v}_{i}, \\
& \frac{d \mathrm{v}_{i}}{d t}=\frac{F_{i}}{m_{d}} ; \quad \text { with } F_{i}=m_{d}\left(\frac{f_{1}}{\tau_{d}}\right)\left(u_{i}-\mathrm{v}_{i}\right), \\
& \frac{d T_{d}}{d t}=\frac{Q+\dot{m}_{d} L_{V}}{m_{d} C_{L}} ; \\
& \frac{d m_{d}}{d t}=\dot{m}_{d}=-m_{d}\left(\frac{1}{\tau_{d}}\right)\left(\frac{\operatorname{Sh}_{3}}{3 \operatorname{Sc}_{G}}\right) \ln \left[1+B_{M}\right],
\end{aligned}
$$

where the subscript $d$ denotes individual droplet conditions, the particle time constant for Stokes flow is $\tau_{d}$ $=\rho_{L} D^{2} /\left(18 \mu_{G}\right), D$ is the droplet diameter, $C_{L}$ is the heat capacity of the liquid, and the latent heat of evaporation is $L_{V}$. Additionally, the gas mixture heat capacity is calculated using a mass averaging; $C_{p, G}=\left(1-Y_{V}\right) C_{p, C}+Y_{V} C_{p, V}$ (evaluated at the droplet location), where $C_{p, C}$ and $C_{p, V}$ are the constant pressure heat capacities of the carrier gas and vapor, respectively $\left(C_{\mathrm{v}, C}\right.$ and $C_{\mathrm{v}, V}$ are the corresponding constant volume heat capacities). The gas phase Prandtl and
Schmidt numbers are $\operatorname{Pr}_{G}=\mu C_{p, G} / \lambda$ and $\mathrm{Sc}_{G}=\mu /(\rho \Gamma)$, respectively. The evaporation rate is driven by the mass transfer number; $B_{M}=\left(Y_{S}-Y_{V}\right) /\left(1-Y_{S}\right)$ (subscript $S$ denotes droplet surface conditions). The semi-empirical RanzMarshall correlations are used for the Nusselt $(\mathrm{Nu})$ and Sherwood (Sh) numbers, whereas $f_{1}$ is an empirical correction to Stokes drag accounting for finite droplet Reynolds numbers $\left[\operatorname{Re}_{d}=\rho\left|u_{i}-\mathrm{v}_{i}\right| D / \mu\right.$ is based on the slip velocity and $\operatorname{Re}_{b}=\rho U_{b} D / \mu$ is based on the blowing velocity, $\left.U_{b}=-\dot{m}_{d} /\left(\pi \rho D^{2}\right)\right]$ :

$$
\begin{aligned}
f_{1}= & \frac{1+0.0545 \mathrm{Re}_{d}+0.1 \operatorname{Re}_{d}^{1 / 2}\left(1-0.03 \mathrm{Re}_{d}\right)}{1+a\left|\operatorname{Re}_{b}\right|^{b}}, \\
a & =0.09+0.077 \exp \left(-0.4 \mathrm{Re}_{d}\right), \\
b & =0.4+0.77 \exp \left(-0.04 \mathrm{Re}_{d}\right) .
\end{aligned}
$$

The function $f_{2}=\beta /\left(e^{\beta}-1\right)$ is an analytical evaporative heat transfer correction, where the nondimensional evaporation parameter $\beta=-1.5 \operatorname{Pr}_{G} \tau_{d} \dot{m}_{d} / m_{d}$ is constant for droplets obeying the " $D^{2}$ law." The vapor surface mass fraction is calculated directly from the surface molar fraction $\left(\chi_{s}\right)$, which is obtained by equating the vapor and liquid fugacities at the surface (i.e., $\chi_{s} P=P_{\text {sat }}$ ), where the saturation pressure $\left(P_{\text {sat }}\right)$ is provided by the Clausius-Clapeyron relation, yielding

$$
\begin{aligned}
Y_{S} & =\frac{\chi_{s}}{\chi_{s}+\left(1-\chi_{s}\right) W_{C} / W_{V}}, \\
\chi_{s} & =\frac{P_{\text {atm }}}{P} \exp \left[\frac{L_{V}}{R_{V}}\left(\frac{1}{T_{B, L}}-\frac{1}{T_{d}}\right)\right],
\end{aligned}
$$

where $P_{\text {atm }}$ is the atmospheric pressure, $T_{B, L}$ is the liquid saturation temperature at $P_{\text {atm }}$ (i.e., the normal boiling temperature). As shown in MBI, the latent heat must be a linear function of temperature for calorically perfect species: $L_{V}$ $=h_{V}^{0}-\left(C_{L}-C_{p, V}\right) T_{d}$.

The choice of a Lagrangian reference frame for the individual droplet conservation equations leads to the following general form for the phase coupling terms:

$S_{I}=-\sum_{a}\left\{\frac{w_{\alpha}}{\Delta x^{3}}\left[\dot{m}_{d}\right]_{\alpha}\right\}$

$S_{\mathrm{II}, i}=-\sum_{\alpha}\left(\frac{w_{\alpha}}{\Delta x^{3}}\left(F_{i}+\dot{m}_{d} \mathrm{v}_{i}\right)_{\alpha}\right)$,

$S_{\mathrm{III}}=-\sum_{\alpha}\left\{\frac{w_{\alpha}}{\Delta x^{3}}\left[\mathrm{v}_{i} F_{i}+Q+\dot{m}_{d}\left(\frac{\mathrm{v}_{i} \mathrm{v}_{i}}{2}+h_{V, S}\right)\right]_{\alpha}\right\}$,

where the summations are over local individual droplet contributions, $h_{V, S}=C_{p, V} T_{d}+h_{V}^{0}$ is the evaporated vapor enthalpy at the droplet surface, and the single droplet evaporation rate $\left(\dot{m}_{d}\right)$, drag force $\left(F_{i}\right)$, and heat transfer rate $(Q)$ are specified by the modeled droplet conservation equations. The local summations are necessarily grid-dependent functions; the summations are over all droplets (subscript $\alpha$ indicates the individual droplet variables; no summation over Greek indices) residing within a local numerical discretization volume $\left(\Delta x^{3}\right)$ and employ a geometrical weighting factor, $w_{\alpha}$, 


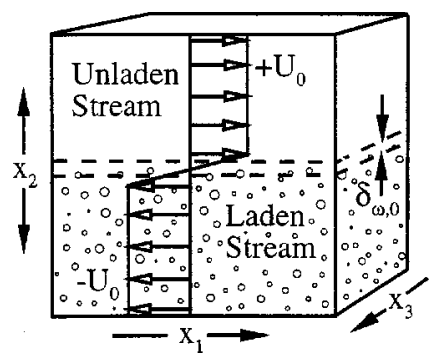

FIG. 1. Schematic of the temporally developing, droplet laden mixing layer.

used to distribute the individual droplet contributions to the eight nearest neighbor surrounding grid points (i.e., corners of the computational volume $\Delta x^{3}$ ). These source terms are then minimally "smoothed" using a conservative operator introduced by Miller and Bellan ${ }^{10}$ in order to retain numerical stability of the Eulerian gas phase fields.

\section{A. Temporally developing mixing layer}

The flow geometry of interest is the temporally developing mixing layer with one droplet laden stream shown in Fig. 1. The streamwise $\left(x_{1}\right)$, cross stream $\left(x_{2}\right)$, and spanwise $\left(x_{3}\right)$ coordinates have lengths $L_{1}, L_{2}$, and $L_{3}$, respectively. Periodic boundary conditions are employed for the $x_{1}$ and $x_{3}$ directions, and adiabatic slip wall conditions are used for the $x_{2}$ boundaries. The initial vorticity thickness is $\delta_{\omega, 0}$, where $\delta_{\omega}(t)=\Delta U_{0} /\left\langle\partial u_{1} / \partial x_{2}\right\rangle_{\max }$; the brackets \langle\rangle indicate averaging over homogeneous $x_{1}-x_{3}$ planes and the mean velocity difference across the layer, $\Delta U_{0}=2 U_{0}$, is calculated from a specified value of the convective Mach number, $M_{c}$ $=U_{0} / \sqrt{R_{c} T_{0} C_{p, C} / C_{\mathrm{v}, C}}\left(T_{0}\right.$ is the initial uniform temperature of the gas). The droplets are initially distributed randomly throughout the $x_{2}<0$ half of the domain with uniform number density and specified size distribution, temperature, and velocity. Finally, the mean velocity and number density profiles are smoothed near the centerline $\left(x_{2}=0\right)$ using an error function profile, and initial forcing perturbations are added to the velocity field in order to excite the growth of both spanwise and streamwise disturbances. ${ }^{10}$

\section{B. Numerical approach}

The governing equations are solved numerically using fourth-order explicit Runge-Kutta temporal integration for all time derivatives and eighth-order accurate central finite differences to represent all spatial derivatives. A fourth-order Lagrange interpolation procedure is used to obtain gas phase variable values at droplet locations, which, in general, do not reside at grid point locations. The droplets are tracked as physical entities (as opposed to stochastic particles used to represent the effects of many "real" particles); i.e., transport equations are solved for every individual droplet in the flow. The approach is essentially the same as that described in MBI with the following three exceptions: First, it was found that artificial numerical oscillations in the solutions can become significant at "long" simulation times. These errors are due primarily to differencing the nonlinear convective terms, similar to aliasing errors in spectral methods. Nondis- sipative central differencing schemes such as those used in this study do not provide numerical diffusion to control the buildup of these artificial oscillations. ${ }^{21}$ In order to alleviate this problem, a tenth-order explicit filtering ${ }^{21}$ is applied to each of the primitive variables at each Runge-Kutta stage (we use only one-tenth of the amplitude suggested in Ref. 21, which was found to provide sufficient dissipation without altering the physical solutions). This filtering was not required in MBI due to the shorter simulation times used in that study. Second, the computational grid is approximately uniformly spaced with constant $\Delta x=\Delta x_{1} \approx \Delta x_{2} \approx \Delta x_{3}$ (these are approximate for reasons described below); whereas MBI used a stretched grid compressed along the $x_{2}=0$ plane.

The third difference from the numerical approach in MBI is that the present simulations are conducted on a Hewlett-Packard Exemplar parallel supercomputer. MBI's FORTAN 77 code has been parallelized using the Message Passing Interface (MPI) subroutines for interprocessor communications. The Eulerian gas phase equations are parallelized using three-dimensional domain decomposition in which all derivatives, source term smoothing and the filtering operation, are performed using the ghost cell methodology. A variety of approaches that differ in their relative levels of communications and load balancing may be employed in parallelizing the Lagrangian droplets. In the present code we choose to treat the droplets by keeping them under the control of the individual processors calculating the local physical space portion of the Eulerian domain in which the droplets reside. This approach has the advantage of minimizing communications costs, but does not load balance well since nearly half of the domain (and processors) has no droplets.

Finally, we note that these simulations are not "pure DNS" in the strict sense due to the fact that some modeling is used even for the gas phase variables in treating the phase coupling terms (see also MBI). There are three primary approximations used in this type of simulation involving the phase coupling: (1) extrapolation of the localized droplet mass, momentum, and energy couplings onto the Eulerian mesh, (2) "smoothing" of the resulting gas phase coupling source terms $\left(S_{\mathrm{I}}, S_{\mathrm{II}, i}\right.$ and $\left.S_{\mathrm{III}}\right)$, and (3) the neglect of perturbations to the velocity field at the droplet scale. Nevertheless, the term DNS has already been adopted in the literature for two-way coupled simulations since the first results for solid particle flows. ${ }^{2}$ Despite these limitations, it is believed that the effects of these approximations on the resulting analyses will be negligible as long as the constraints on the droplet scales necessary for the derivation of the governing equations are met (see below). Furthermore, all simulations are performed with substantially larger than necessary resolutions (for comparable single phase flows) in order to ensure the accuracy of the results.

\section{RESULTS}

All simulations are conducted within a domain having $L_{1}=4 \lambda_{1}=29.16 \delta_{\omega, 0}, \quad L_{2}=1.1 L_{1}$, and $L_{3}=4 \lambda_{3}=0.6 L_{1}$, where $\lambda_{1}$ and $\lambda_{3}$ are the forcing wavelengths in the $x_{1}$ and $x_{3}$ directions, respectively (the code is written in dimensional variables using $L_{1}=0.2 \mathrm{~m}$ ). This allows for the instigation of 
TABLE I. Property values used in the simulations. The subscripts $C$ and $V$ denote the carrier gas and evaporated vapor, respectively.

\begin{tabular}{cl}
\hline \hline Property & \multicolumn{1}{c}{ Value } \\
\hline$W_{C}$ & $28.97 \mathrm{~kg}(\mathrm{~kg} \cdot \mathrm{mole})^{-1}$ \\
$W_{V}$ & $142.0 \mathrm{~kg}\left(\mathrm{~kg} \cdot \mathrm{mole}^{-1}\right.$ \\
$C_{p, C}$ & $1004.8 \mathrm{~J} \mathrm{~kg} \mathrm{~K}^{-1}$ \\
$C_{p, V}$ & $1939.6 \mathrm{~J} \mathrm{~kg}^{-1} \mathrm{~K}^{-1}$ \\
$C_{L}$ & $2520.5 \mathrm{~J} \mathrm{~kg}$ \\
$\operatorname{Pr}_{G}$ & 0.697 \\
$\rho_{L}$ & $642 \mathrm{~kg} \mathrm{~m}^{-3}$ \\
$T_{B, L}$ & $447.7 \mathrm{~K}$ \\
$h_{V}^{0}$ & $5.35 \times 10^{5} \mathrm{~J} \mathrm{~K}^{-1}$ \\
\hline \hline
\end{tabular}

four spanwise rollers along with both first and second subharmonics (with amplitudes 0.5 and 0.35 relative to the primary disturbance) to excite multiple pairing events. The forcing amplitudes are $F_{2 D}=10 \%$ and $F_{3 D}=2.25 \%$ relative to the respective spanwise and streamwise circulations. ${ }^{10}$ The convective Mach number is $M_{c}=0.35$, the initial gas temperature is $T_{0}=375 \mathrm{~K}$, and the pressure is initially uniform and equal to atmospheric pressure, $P_{0}=P_{\text {atm }}$. The droplets are initially randomly dispersed throughout the $x_{2} \leqslant 0$ portion of the domain having uniform number density, zero local slip velocity, and uniform temperature $T_{d, 0}=345 \mathrm{~K}$. In contrast to MBI, we consider polydisperse initial droplet distributions: The droplet time constants are chosen using a random number generator such that the Stokes number, $\mathrm{St}$ $=\tau_{d} \Delta U_{0} / \delta_{\omega, 0}$, has a Gaussian distribution (truncated to \pm three standard deviations) at each location with $x_{2} \leqslant 0$, with a mean $\langle\mathrm{St}\rangle=3$ and standard deviation $\left\langle(\mathrm{St}-\langle\mathrm{St}\rangle)^{2}\right\rangle^{1 / 2}=\frac{1}{2}$. The two calorically perfect species considered have properties corresponding to those of air for the carrier gas and decane for the liquid/vapor (Table I). These were referred to as pseudoair and pseudodecane in MBI because the viscosity, thermal conductivity, and species diffusivity have artificially large values in order to enable a complete resolution of all pertinent length scales as required in DNS (for single-phase flows). Finally, the initial gas phase is composed entirely of the pure carrier gas.

The simulations conducted for the present study are summarized in Table II, which provides the flow initial Reynolds number $\left(\operatorname{Re}_{0}=\rho_{0} \Delta U_{0} \delta_{\omega, 0} / \mu\right)$, the grid resolution, total number of droplets tracked, the mass loading ratio ML (mass of liquid divided by mass of gas for $x_{2} \leqslant 0$ ), mean droplet number density (per unit $\delta_{\omega, 0}^{3}$ volume), and the grid spacing and time step relative to corresponding droplet scales. Each of the runs described in Table II is named for both the type of flow (SP for single phase and TP for two phase) and the flow initial Reynolds number. The droplet volume fraction (VF) must be negligible ( $\sim 10^{-3}$ or smaller) in order to satisfy the assumptions made in deriving the governing equations. Evaluation of VF [which is related to the mass loading through the ratio of densities $\left.\left(\rho / \rho_{L}\right)\right]$ yields $\mathrm{VF}=2.9$ $\times 10^{-4}$ and $\mathrm{VF}=7.2 \times 10^{-4}$ for $\mathrm{ML}=0.2$ and $\mathrm{ML}=0.5$, respectively. By fixing the remaining flow parameters, the simulations described in Table II allow us to study the isolated effects of the flow initial Reynolds number at fixed $\mathrm{ML}=0.2$ (runs TP200, TP350, TP500a, and TP600) as well
TABLE II. Simulation parameters ( $\mathrm{SP}=$ single phase, $\mathrm{TP}=$ two-phase) including the Reynolds number, grid resolution, number of droplets, mass loading ratio, number density $\left(n^{*}\right.$ is the number of droplets per unit $\delta_{\omega, 0}^{3}$ volume), and relative droplet length and time scales: All runs are initialized with $M_{c}=0.35, T=375 \mathrm{~K}$ and $P=1$ atm. TP runs are initialized with $\mathrm{V}_{i}$ $=u_{i}, T_{d}=345 \mathrm{~K}$ and polydisperse Gaussian $\tau_{d}$ distributions such that $\left\langle\mathrm{St}_{0}\right\rangle=3$ and $\mathrm{St}_{0, \mathrm{rms}}=0.5$.

\begin{tabular}{cccccccc}
\hline \hline Run & $\operatorname{Re}_{0}$ & $N_{1} \times N_{2} \times N_{3}$ & $N_{D}$ & $\mathrm{ML}$ & $n^{*}$ & $\Delta x /\left\langle D_{0}\right\rangle$ & $\left\langle\tau_{d, 0}\right\rangle / \Delta t$ \\
\hline $\mathrm{SP} 200$ & 200 & $100 \times 112 \times 60$ & 0 & $\cdots$ & $\cdots$ & $\cdots$ & $\ldots$ \\
$\mathrm{TP} 200$ & 200 & $100 \times 112 \times 60$ & $5.8 \times 10^{5}$ & 0.2 & 71 & 14.7 & 20.5 \\
$\mathrm{TP} 350$ & 350 & $168 \times 184 \times 100$ & $1.3 \times 10^{6}$ & 0.2 & 159 & 11.58 & 33.3 \\
SP500 & 500 & $252 \times 276 \times 152$ & 0 & $\cdots$ & $\cdots$ & $\ldots$ & $\ldots$ \\
TP500a & 500 & $252 \times 276 \times 152$ & $2.3 \times 10^{6}$ & 0.2 & 281 & 9.22 & 50.0 \\
TP500b & 500 & $252 \times 276 \times 152$ & $5.7 \times 10^{6}$ & 0.5 & 697 & 9.22 & 50.0 \\
TP600 & 600 & $300 \times 332 \times 180$ & $3.0 \times 10^{6}$ & 0.2 & 367 & 8.49 & 61.4 \\
\hline \hline
\end{tabular}

as the effects of the mass loading ratio for fixed $\mathrm{Re}_{0}=500$ (runs SP500, TP500a, and TP500b). Note also that the grid spacings in each direction $\left(\Delta x_{1}, \Delta x_{2}, \Delta x_{3}\right)$ are not always exactly equal since the number of grid points must be evenly divisible by the number of processors used in each direction. Unless otherwise stated, all of the simulations were performed using 64 processors with a $4 \times 4 \times 4$ spatial decomposition.

The simulations described below differ from those in MBI in several ways. The gas temperature is higher here in order to induce stronger evaporation rates and delay the flow saturation process. ${ }^{10} \mathrm{We}$ also consider here polydisperse rather than monodisperse droplet size distributions in order to produce a more "natural" two-phase flow. Note that the Gaussian Stokes number (i.e., $D^{2}$ ) distribution produces a diameter distribution which is skewed toward smaller droplets in accordance with experimental observations of sprays. $^{22}$ However, the most important difference in the present simulations is the combination of higher Reynolds numbers and multiple spanwise vortex pairing excitations. In MBI only pretransitional mixing was considered for shear layers dominated primarily by large-scale spanwise and streamwise vortical structures. The present flow simulations are performed in order to capture the transition to turbulence (described in detail below) and create a more "natural", flow field indicative of higher Reynolds number experimental flows. Moser and Rogers ${ }^{23,24}$ showed that the temporally developing mixing layer will evolve through a natural transition to small-scale mixing turbulence at sufficiently large Reynolds numbers when multiple pairings and substantial streamwise forcing perturbations are present. Their simulations were conducted for incompressible, single-phase Navier-Stokes flows using a pseudospectral numerical method and resolutions as large as $192 \times 212 \times 128$. Simulation SP500 in Table II is nearly identical to simulations HIGH2P and TURB2P in Ref. 24 (we do not begin with a turbulent field as in TURB2P, but we break the natural symmetry between streamwise roller pairs, which was retained in HIGH2P), with the primary exceptions that SP500 is compressible flow with confined boundary conditions rather than infinite free streams. Despite these similarities, simulation SP500 uses a substantially finer grid resolution for two rea- 
TABLE III. The total memory requirement in gigabytes, average clock time per iteration $\left(t_{c}\right)$ running on 64 (CPUs) (* indicates times on 8 CPUs), and the total number of iterations $\left(N_{\text {its }}\right)$ for each simulation.

\begin{tabular}{cccc}
\hline \hline Run & Memory $(\mathrm{GB})$ & $t_{c}(\mathrm{~s})$ & $N_{\mathrm{its}}$ \\
\hline SP200 & 0.356 & $1.09(10.4)^{*}$ & 711 \\
TP200 & 0.844 & $4.45(31.6)^{*}$ & 643 \\
TP350 & 2.54 & 15.1 & 1175 \\
SP500 & 3.74 & 42.4 & 1835 \\
TP500a & 6.68 & 57.0 & 1799 \\
TP500b & 9.30 & 82.6 & 1772 \\
TP600 & 10.8 & 102.5 & 2205 \\
\hline \hline
\end{tabular}

sons: First, the present numerical method, though of highorder accuracy, is not as accurate as the pseudospectral technique used by Moser and Rogers; and second, a finer resolution is used to ensure an accurate capture of the twophase flows TP500a and TP500b which contain additional two-way coupling effects on the small scales of the gas phase. Note that our simulation TP600 has a flow initial Reynolds number that is $20 \%$ larger than the largest simulated by Moser and Rogers. ${ }^{24}$ In contrast, the largest temporally developing mixing layer simulations performed to date were initialized using two velocity fields obtained from separate DNS of a turbulent boundary layer in order to produce a more natural turbulence and self-similar states. ${ }^{25}$ These simulations were also for single-phase incompressible flow and utilized as many as $512 \times 210 \times 192$ collocation points $(\approx 15 \%$ more grid points than TP600). We do not have turbulent droplet laden boundary layer simulations available and therefore choose to study the transition process from initially laminar (though perturbed) velocity fields. Finally, we note that the present simulations, while rivaling the largest previous single-phase DNS resolutions, employ nearly an order of magnitude more Eulerian grid points, and nearly a factor of 6 more discrete particles than previous two-phase DNS.

Table III shows the total memory, average clock time per iteration (on both 8 and $64 \mathrm{CPUs}$ ), and the total number of iterations for all of the simulations. The iteration time $t_{c}$ is an average over all iterations and is based on the clock time rather than the CPU time, which is unavailable on this architecture. Therefore, $t_{c}$ can fluctuate significantly from one simulation to the next due to the relative level of computer usage at the time the simulations are performed. Nevertheless, the scale-up is nearly linear with both memory size and number of CPUs for both single-phase and two-phase simulations (not shown graphically). Finally, Table IV shows the relative time spent on each portion of the droplet calculations

TABLE IV. The approximate relative time spent on the various droplet operations for the two-phase flow simulation TP200 (64 CPUs).

\begin{tabular}{lc}
\hline \hline \multicolumn{1}{c}{ Calculation } & Simulation time \\
\hline Interpolation & $40 \%$ \\
Source term calculation & $12 \%$ \\
Source term smoothing & $12 \%$ \\
Droplet transfer & $36 \%$ \\
\hline \hline
\end{tabular}

including the interpolation, building source terms $\left(S_{\mathrm{I}}, S_{\mathrm{II}, i}\right.$, and $\left.S_{\mathrm{III}}\right)$, source term smoothing and also the droplet transfer between CPUs as the droplet enter/exit the portions of the domain controlled by the processors. Each of these procedures must be performed during each Runge-Kutta stage (four times per time step). The table clearly shows the relatively heavy toll that droplet communication plays in a mixed Eulerian/Lagrangian flow problem such as this, despite minimizing communication costs at the expense of load balancing.

\section{A. Evolution of the mixing layer}

The mixing layers described in this paper have many similarities to those described in MBI. In order to avoid excessive repetition of known results we first give a brief summary of some of the salient features of droplet laden mixing layers as observed in MBI. In that study, Miller and Bellan investigated the effects of the mass loading ratio, the initial droplet size, the streamwise forcing amplitude, and the initial droplet temperature (for $\mathrm{Re}_{0}=200$ with a single spanwise vortex pairing). They found that for the low gas temperature mixing layers, all of the ML $>0.02$ flows underwent a process of "evaporative saturation." For these flows, the droplet laden stream rapidly became saturated (a cessation of vaporization) due to a combined buildup of free stream evaporated vapor and cooling of the gas flow due to latent heat effects. It was also observed that this saturated state (described by the long time mean gas temperature and vapor mass fraction profiles) is essentially independent of the initial droplet mass loading ratio and size distributions; i.e., it is primarily governed by the initial gas temperature and the latent heat of the liquid. Thereafter, the mixing layer behaved as a density stratified (due to the evaporation) mixing layer in which the laden stream contained solid (nonevaporating) particles; with the exception being that droplets entrained into the mixing region are in contact with higher-temperature, lower vapor content, fluid and can continue to completely vaporize. In MBI this saturated state was characterized by mean temperatures and vapor mass fractions in the laden stream of $\langle T\rangle$ $\approx 340 \mathrm{~K}$ (with $T_{0}=350 \mathrm{~K}$ ) and $\left\langle Y_{V}\right\rangle \approx 0.04$. In the present simulations a similar saturation process is also observed; however, the initial gas temperature is larger, so that the flows saturate with $\langle T\rangle \approx 345 \mathrm{~K}$ and $\left\langle Y_{V}\right\rangle \approx 0.09$ (not shown). The differences between the present flows and those in MBI are therefore primarily in the excitation of small scales inherent to the mixing transition process. Therefore, only a relatively brief description of the physical evolution of the flows is given below prior to presenting the subgrid analyses, which are the main focus of this study.

One of the most fundamental measures of the development of a mixing layer is its growth rate. The growth rate is generally measured by examining the time evolution of a mixing layer thickness scale such as that given by the vorticity thickness. However, the vorticity thickness is a function of the mean velocity derivative and can be sporadic in time. Therefore, a smoother measure of the growth rate is given by examining an integral quantity such as the momentum thickness denoted by $\delta_{m}$. Previously, $\delta_{m}$ has generally 


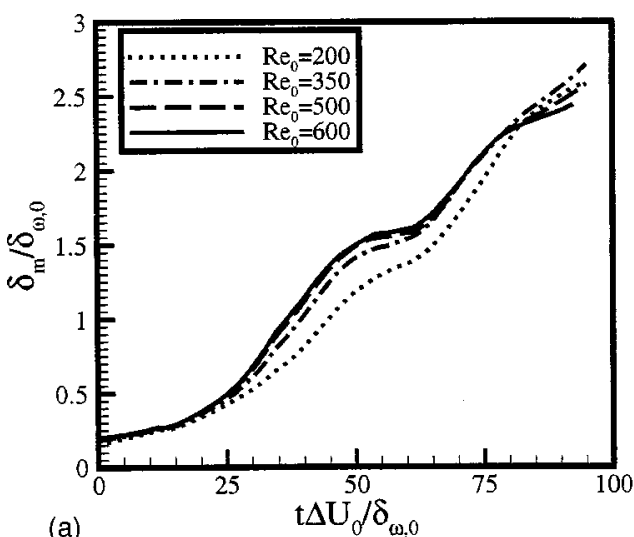

(a)

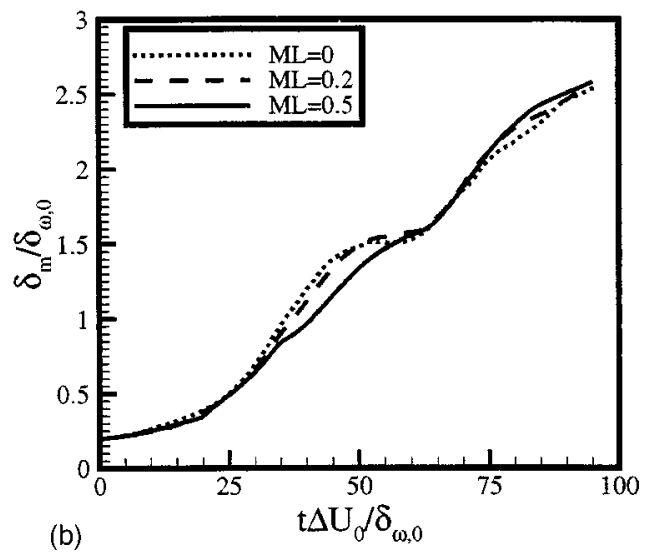

FIG. 2. Temporal development of the momentum thickness normalized by the initial vorticity thickness as a function of the (a) initial Reynolds number at fixed $\mathrm{ML}=0.2$, and (b) mass loading ratio at fixed $\mathrm{Re}_{0}=500$.

been defined to describe either constant density, or timeindependent free stream momentum flows. ${ }^{24}$ However, the present flow is characterized by a time-dependent mean gas phase momentum in the laden stream due to the evaporation of the high molecular weight vapor. Therefore, we define a new momentum thickness as

$$
\begin{aligned}
\delta_{m}= & \frac{1}{\left[\left\langle\rho u_{1}\right\rangle_{2}-\left\langle\rho u_{1}\right\rangle_{1}\right]^{2}} \int_{-L_{2} / 2}^{+L_{2} / 2}\left\{\left[\left\langle\rho u_{1}\right\rangle_{2}-\left\langle\rho u_{1}\right\rangle\left(x_{2}\right)\right]\right. \\
& \left.\times\left[\left\langle\rho u_{1}\right\rangle\left(x_{2}\right)-\left\langle\rho u_{1}\right\rangle_{1}\right]\right\} d x_{2}
\end{aligned}
$$

where $\left\langle\rho u_{1}\right\rangle_{1}$ and $\left\langle\rho u_{1}\right\rangle_{2}$ are the time-dependent, planaraveraged streamwise momenta evaluated at the $x_{2}=-L_{2} / 2$ and $x_{2}=+L_{2} / 2$ slip walls, respectively (note that $\left\langle\rho u_{1}\right\rangle_{1}$ is negative). This definition accounts for the effects of evaporation, as well as the finite bounded domain size and recovers previous expressions when the density is constant and the walls are moved infinitely far apart. Figure 2 depicts the temporal evolution of the momentum thickness as a function of both the flow Reynolds number and the mass loading ratio. It is known from previous single-phase DNS studies that the effects of forcing can significantly influence the entire duration of a mixing layer, and "truly", self-similar profiles are only obtained when unforced, turbulent initial conditions are used ${ }^{25}$ (see also Ref. 26); in which case linear growth rates would be expected. The oscillations in the momentum thickness curves in Fig. 2 are therefore evidence of forcing influence on the mixing layer development. As the flow Reynolds number is increased, the corresponding decrease in viscosity results in a growth rate that is more strongly influenced by the initial forcing, as evidenced by the more discernible leveling of the curves during the two pairing events (at times $t \Delta U_{0} / \delta_{\omega, 0} \approx 45$ and $\approx 80$ ). Figure $2(\mathrm{~b})$ illustrates the effects of the mass loading ratio on $\delta_{m}$. Similar to MBI, we observe a reduction in the growth rate with increased ML through the time of the first pairing event (the simulations in MBI ceased at time $\left.t \Delta U_{0} / \delta_{\omega, 0}=50\right)$. However, after this time the trend reverses and the second pairing culminates with case $\mathrm{ML}=0(\mathrm{SP} 500)$ having the smallest momentum thickness. This feature can be attributed to the presence of the droplets acting to reduce the influence of the initial forcing, as indicated by the more linear nature of the curves in Fig. 2(b) with increasing ML (explained below). It would appear that the growth rates for all curves in Fig. 2(b) are essentially the same; however, lower mass loading runs show stronger influence of the forcing (indicated by the leveling at the pairing times) and therefore tend to "crisscross" the more linear large loading curves. In this respect, the conclusion in MBI regarding the effects of ML on the mixing layer growth rate applies only to the single-pairing, pre-transitional simulations conducted in that work and not to the post-transitional state of the layer. We incidentally note that the momentum thickness Reynolds number $\left(\operatorname{Re}_{m}\right.$ $\left.=\rho_{0} \Delta U_{0} \delta_{m} / \mu\right)$ commonly used to describe mixing layers can be obtained by multiplying the curves in Fig. 2 by their corresponding $\mathrm{Re}_{0}$ values: For example, simulation TP600 has $\mathrm{Re}_{m} \cong 1460$ at the final time.

The temporal development of two indicators of the transition to mixing turbulence are presented in Fig. 3. The first is the instantaneous squared vorticity magnitude averaged over the entire domain (denoted by the double brackets, «»). The second indicator is the average of the positive portion of the squared spanwise vorticity magnitude normalized by the total average of the same squared component [including the mean vorticity; $H$ in Figs. 3(c) and 3(d) is the Heaviside function]. These averages provide a good indicator of the rotational nature of the flow fields at all times, since the use of adiabatic slip-wall/periodic boundary conditions implies that a fixed amount of fluid mass and total energy are being tracked within the system. Moser and Rogers $^{24}$ showed that transition begins during the first pairing event for sufficiently three-dimensional flows as a result of interactions between "collapsed rib" and "cup" structures. This process is indicated quantitatively in Figs. 3(a) and 3 (b) by the sudden rapid increase in the rotational energy of the fluid within the domain in the time interval between the first rollup $\left(t \Delta U_{0} / \delta_{\omega, 0} \approx 25\right)$ and the completion of the first pairing $\left(t \Delta U_{0} / \delta_{\omega, 0} \approx 50\right)$ for sufficiently large $\mathrm{Re}_{0}$. According to Moser and Rogers, after the first pairing, vortex stretching increases the complexity of the flow until the second pairing produces an apparently fully turbulent flow. This description of the process is consistent with observations of the present droplet laden flows (see also below). The relative amount of positively rotational fluid [see Figs. 3(c) and 3(d)] is also particularly indicative of the transition to turbulence, since the mean flow is entirely composed of $\omega_{3} \leqslant 0$ at time 

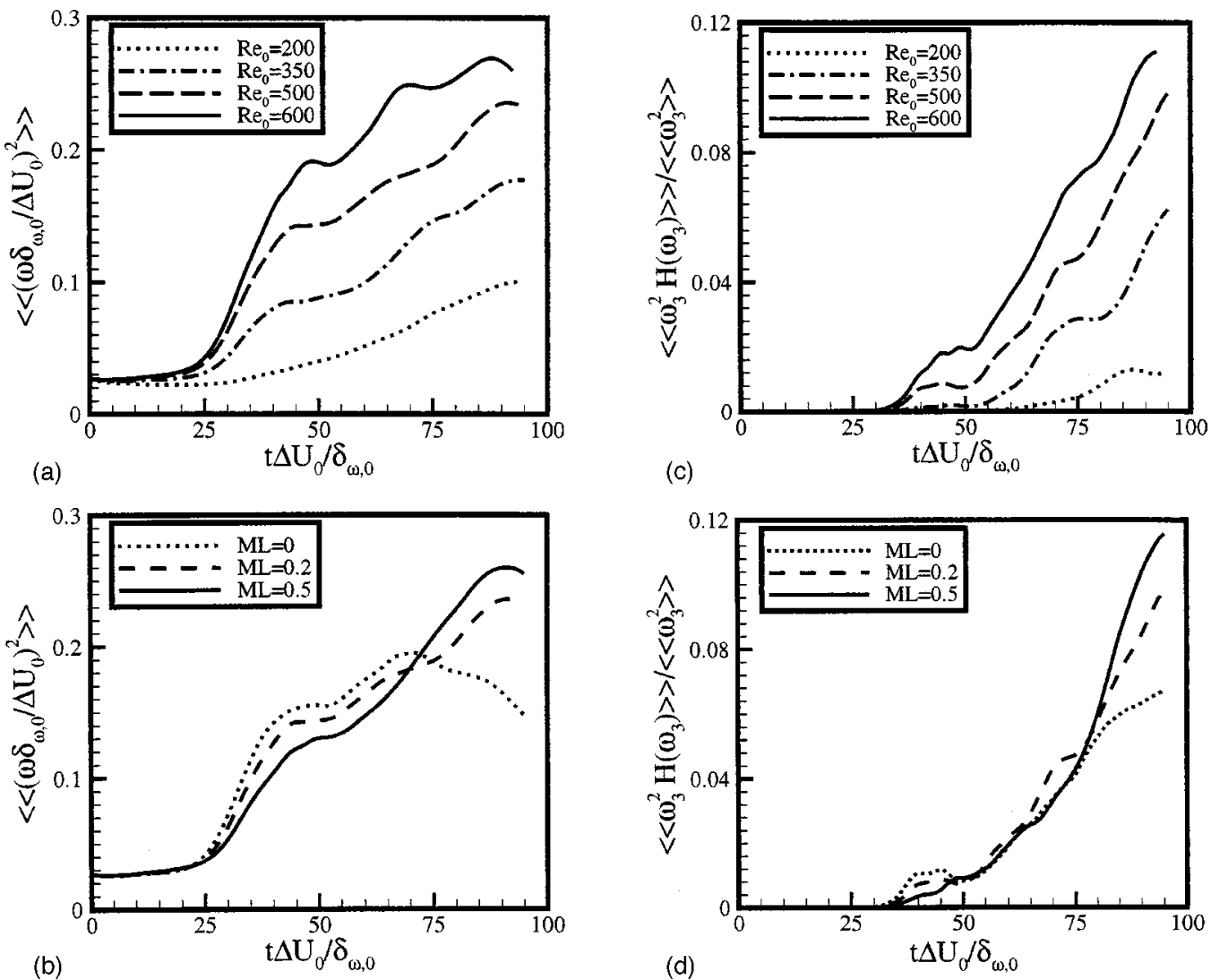

FIG. 3. Temporal development of normalized quantities integrated over the domain. (a) Vorticity magnitude for $\mathrm{ML}=0.2$, (b) vorticity magnitude for Re 0 $=500$, (c) relative positive spanwise vorticity for $M L=0.2$, and (d) relative positive spanwise vorticity for $\operatorname{Re}_{0}=500$.

$t=0$. Therefore, any positive vorticity appearing at later times must be produced by vortex stretching and bending, which produces small-scale "eddies" of both signs. ${ }^{24}$ Clearly, the degree of transition increases markedly with $\mathrm{Re}_{0}$; however, it is interesting to note that the flow is more rotational for large mass loading ratios [see also Fig. 3(b)]. The increase of rotational fluid energy with ML occurs, despite the fact that, on average, the flow loses kinetic energy to the droplets through the drag force. ${ }^{10}$ This effect can presumably be attributed to two influences. First, the curl of the momentum source term, $\nabla \times \overrightarrow{S_{\text {II }}}$, may act directly as a production mechanism for the vorticity equation (note that calculating the actual curl numerically is not feasible due to the spatially intermittent nature of the coupling source terms). Second, the source term $S_{\mathrm{II}, i}$ is composed of a variety of wavelengths that can introduce disturbances in addition to those intentionally installed by the forcing.

\section{Instantaneous flow fields}

The qualitative nature of the fully developed flow fields is illustrated in Figs. 4 and 5, which show instantaneous contours at time $t \Delta U_{0} / \delta_{\omega, 0}=85$ from cases TP600 and TP500b, respectively. At this time, the corresponding momentum thickness Reynolds numbers are $\operatorname{Re}_{m}=1400$ and $\mathrm{Re}_{m}=1213$ for runs TP600 and TP500b, respectively. The contours correspond to the same "rib plane" (a plane intersecting one of the initially forced streamwise vortices), and depict the spanwise vorticity, vapor mass fraction, and drop- let number density. The number density is calculated exactly as the coupling source terms described above, i.e., $n$ $=\Sigma_{\alpha}\left\{w_{\alpha} / \Delta x^{3}\right\}$, and has dimensions of number of droplets per unit volume. Contours of $n$ are only qualitative representations of the relative droplet locations within the particular planes, as $n$ is a grid-dependent function with little quantitative value. ${ }^{10}$ Note also that the contours of $n$ only provide information on the droplet locations, not the droplet sizes and/or degree of evaporation. Despite the discernible presence of the large-scale structures, both the TP600 and TP600b flows exhibit a broad range of excited eddy scales and are qualitatively similar to experimentally observed turbulent mixing layers. In addition to the obviously more "turbulent" vorticity and scalar fields, the droplet concentration fields are in both cases significantly different than those described in MBI. In that pretransitional study, the droplets were shown to be "thrown" from the high-vorticity fluid and to congregate in high strain regions due to their inertia through the preferential concentration mechanism, ${ }^{27}$ the droplet locations "'mapped" both the primary spanwise and streamwise structures by clearly clumping the droplets around the peripheries of both structures (the "focusing" effect discussed by Crowe et al. ${ }^{28}$ ). Although the number density contours in Figs. 4(c) and 5(c) are still indicative of preferential concentration, this occurs on a much smaller scale than those in MBI. Particularly for simulation TP600, with its larger Reynolds number, the concentration field shows a mix of characteristics seen in both MBI and those 

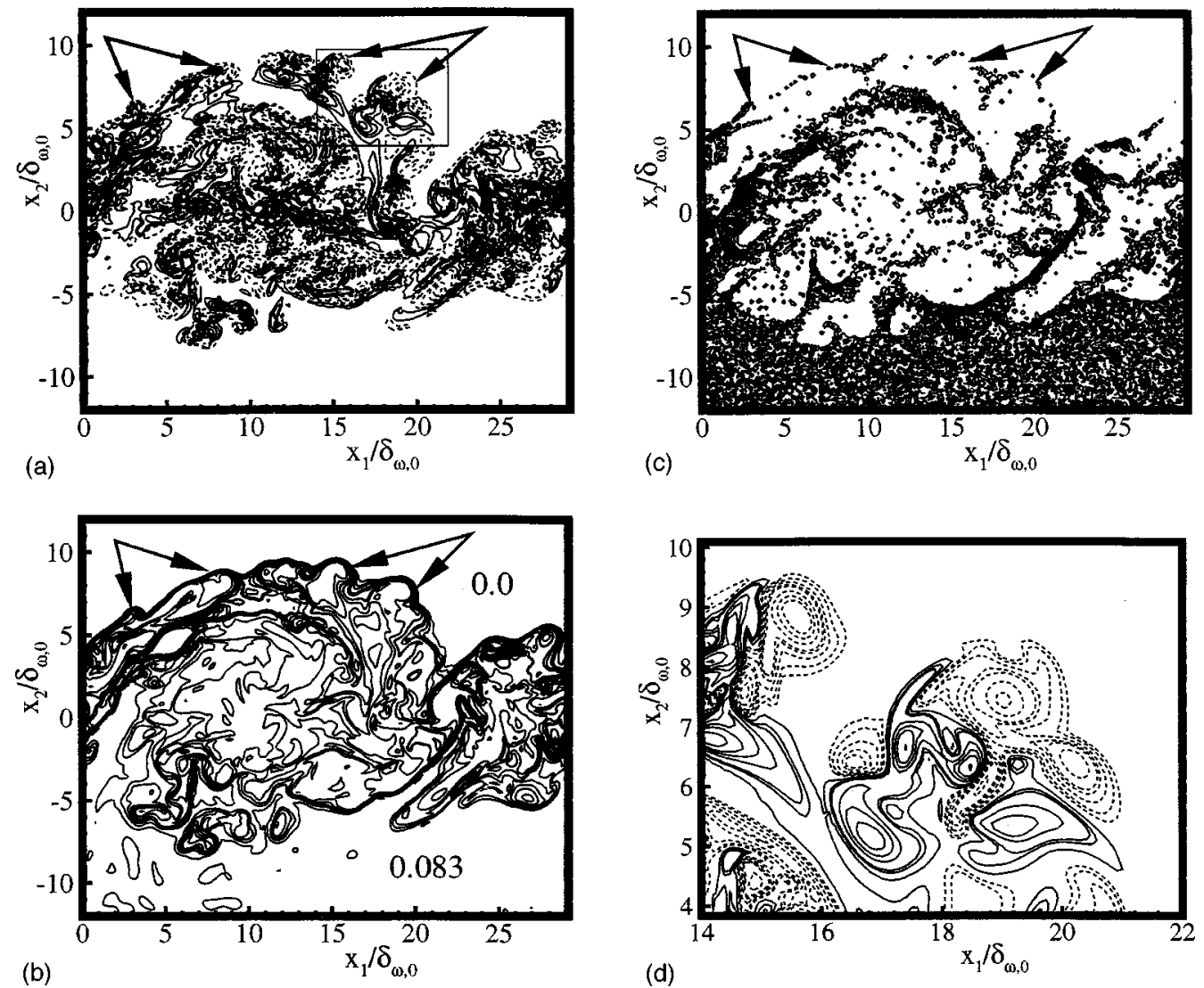

FIG. 4. Instantaneous contours at time $t \Delta U_{0} / \delta_{\omega, 0}=85$ from simulation TP600 $\left(\operatorname{Re}_{0}=600, \mathrm{ML}=0.2 ; \operatorname{Re}_{m}=1400\right)$ in a rib plane $\left(x_{3}=3 \lambda_{3} / 4\right)$. (a) Spanwise vorticity $\omega_{3}$ (dashed lines for negative values), (b) vapor mass fraction $Y_{V}$, (c) droplet concentration, and (d) blow up of the boxed region in part (a) also showing $\omega_{3}$ contours.

observed previously in DNS of solid particle dispersion in isotropic turbulence. ${ }^{27}$ These observations of smaller-scale preferential concentration in the mixing layer are in agreement with experimental observations of high Reynolds number particle laden mixing layers (e.g., Ref. 29). Remarkably, although the TP500b flow field shown in Fig. 5 has a lower $\mathrm{Re}_{0}$ than TP600, the large-scale structure appears to be less organized, and correspondingly more characteristic of "real" turbulent mixing layers. This observation is consistent with the previously shown momentum thickness in that the larger mass loading ratios appear to "break up" the effects of the initial forcing perturbations, thus resulting in a more "natural" turbulence. Note that the droplet concentration appears to be somewhat more organized within the mixing layer for the $\mathrm{ML}=0.5$ case [Fig. 5(c)] as compared to the $\mathrm{ML}=0.2$ flow at the same $\operatorname{Re}_{0}$ (Fig. 6). This is evidenced by the appearance of some droplets within the "void" regions of vortical fluid in the interior of the layer, and occurs because the droplets in Fig. 6 are smaller than those for ML $=0.5$ (see below) due to a relatively earlier saturation of the larger mass loading flows (see also MBI).

Moser and Rogers ${ }^{24}$ discussed the presence of secondary Kelvin-Helmholtz-type rollup within their largest Reynolds number mixing layer simulations, resulting in the formation of thin sheets of spanwise vorticity (see, e.g., Fig. 5 of the citation). These secondary rollups were observed along the interior and periphery of the primary spanwise vortices and are considered to aid in the transition to turbulence. Similar features are observed in the present high Reynolds number simulations (SP500, TP500a, TP500b, and TP600; not shown), but, in addition, and only for the highest Reynolds number case, TP600, we find an additional, though similar, Kelvin-Helmholtz-type rollup located along the edge of the braids. This instability is indicated by the arrows in Fig. 4 and is most clearly discernible in the vapor mass fraction contours of Fig. 4(b). The mean flow is to the right in the upper portion of the figures $\left(x_{2}>0\right)$, creating a shearing instability beginning in the braid region of the upper left quadrant of the figures. Small Kelvin-Helmholtz instabilities are clearly visible on the free stream edge of the braid in the contours. As the mean free stream flow passes over the braid, the associated shearing causes the instabilities to travel toward the center of the domain, growing in size (additional arrows). The turbulent/laminar boundary on the upper portion of the mixing layer therefore acts very much like a spatially developing mixing layer of smaller scale than the primary layer on which it is formed. The third and fourth arrows to the right of Fig. 4(b) clearly show these secondary vortices as discerned by the vapor mass fraction contours. The $\omega_{3}$ contours within the boxed section in Fig. 4(b) highlighted in Fig. 4(d) present evidence of an interaction or pairing of these secondary structures [note that $\delta_{\omega, 0} / \Delta x$ $\approx 10.3$, so Fig. 4(d) contains approximately $82 \times 62$ grid points]. Finally, as the primary mixing layer continues to 

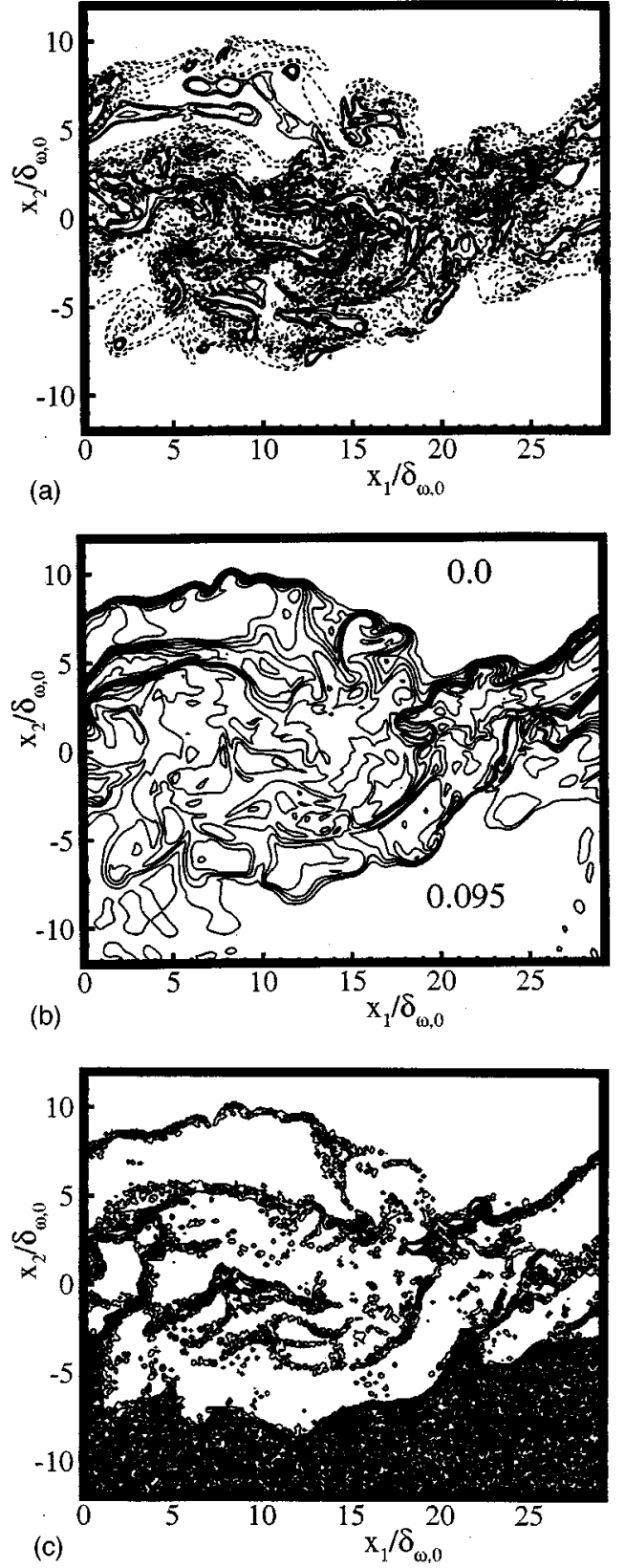

FIG. 5. Instantaneous contours at time $t \Delta U_{0} / \delta_{\omega, 0}=85$ from simulation TP500b $\left(\operatorname{Re}_{0}=500, \mathrm{ML}=0.5 ; \operatorname{Re}_{m}=1213\right)$ in a rib plane $\left(x_{3}=3 \lambda_{3} / 4\right)$. (a) Spanwise vorticity $\omega_{3}$ (dashed lines for negative values), (b) vapor mass fraction $Y_{V}$, and (c) droplet concentration.

grow, these structures are engulfed into the larger-scale spanwise structure increasing the turbulent nature of the flow. These secondary features are not observed either for the lower Reynolds number flows with $\mathrm{Re}_{0}=500$, nor on the $x_{2}$ $<0$ portion of the TP600 flow. This lack of secondary rollups on the laden stream side of the layer can be attributed to the stabilizing nature of the larger density gradients in this region (not shown) that result from the evaporation of the high molecular weight droplets (see also MBI). We therefore conclude that the observed secondary instability is a high Reynolds number effect and should be prevalent in both single-phase and multiphase experimental turbulent mixing layers.

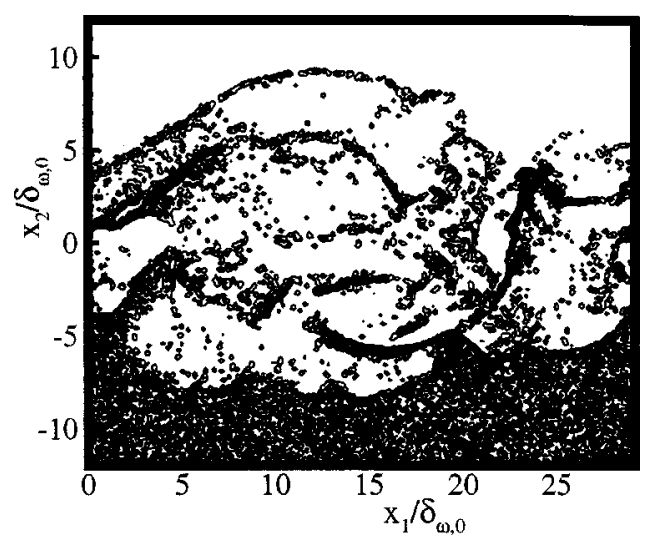

FIG. 6. Instantaneous contours of the droplet concentration at time $t \Delta U_{0} / \delta_{\omega, 0}=85$ from simulation TP500a $\quad\left(\operatorname{Re}_{0}=500, \quad \mathrm{ML}=0.2 ; \quad \operatorname{Re}_{m}\right.$ $=1179)$ in a rib plane $\left(x_{3}=3 \lambda_{3} / 4\right)$.

\section{Droplet size distributions}

As mentioned above, the simulations in Table II were all initialized with polydisperse droplet size distributions. Each of the flows has an initially uniform number density in the laden stream; however, the local droplet sizes are chosen randomly such that the resulting Stokes number distribution is Gaussian with $\langle\mathrm{St}\rangle=3$ and standard deviation equal to 0.5. Figure 7 shows the long time statistical state of the Stokes number distribution for each of the two-phase flow simulations. At this time the layer is quasi-saturated with evaporation occurring primarily only in the interior of the mixing region. These statistics, and all following planar-averaged droplet statistics, are calculated by dividing the $x_{2}$ domain coordinate into 40 equal-sized bins and calculating averages over all droplets residing within each bin. In order to achieve accurate statistics, only bins containing greater than $10^{3}$ droplets are considered. By definition, the normalized third and fourth moments for any variable $\phi$ are the skewness factor $\left(\mu_{3}\right)$ and the flatness factor $\left(\mu_{4}\right)$, defined by

$\mu_{3}(\phi)=\frac{\left\langle(\phi-\langle\phi\rangle)^{3}\right\rangle}{\left\langle(\phi-\langle\phi\rangle)^{2}\right\rangle^{3 / 2}}, \quad \mu_{4}(\phi)=\frac{\left\langle(\phi-\langle\phi\rangle)^{4}\right\rangle}{\left\langle(\phi-\langle\phi\rangle)^{2}\right\rangle^{2}}$.

For the initial Stokes number probability density function (PDF), or for any Gaussian PDF, $\mu_{3}=0$ and $\mu_{4}=3$. The illustrations of Figs. 7(a)-7(d) show that the ultimate state of the Stokes number distribution is nearly independent of $\mathrm{Re}_{0}$ over the range considered, and is only a function of ML. The reduction in the initial value of $\langle\mathrm{St}\rangle$ is due to evaporation and is therefore an indication of the statistical state of the saturation process (i.e., lower $\langle\mathrm{St}\rangle$ indicates later saturation with respect to the initial total liquid mass). It is also interesting to note that the Stokes number standard deviation does not change in the laden stream, despite the evaporation. This presumably occurs because the temperature (of both the drops and the gas) is relatively uniform and the evaporation rate is relatively slow [see Fig. 10(b)], which results in the deviations from the initial variance of the distribution being small. On the other hand, interior to the layer the standard deviation actually increases from its initial value of 0.5 ; we conclude that the turbulence acts to increase the relative range of droplet sizes. For all five flows of Fig. 7, the Stokes 

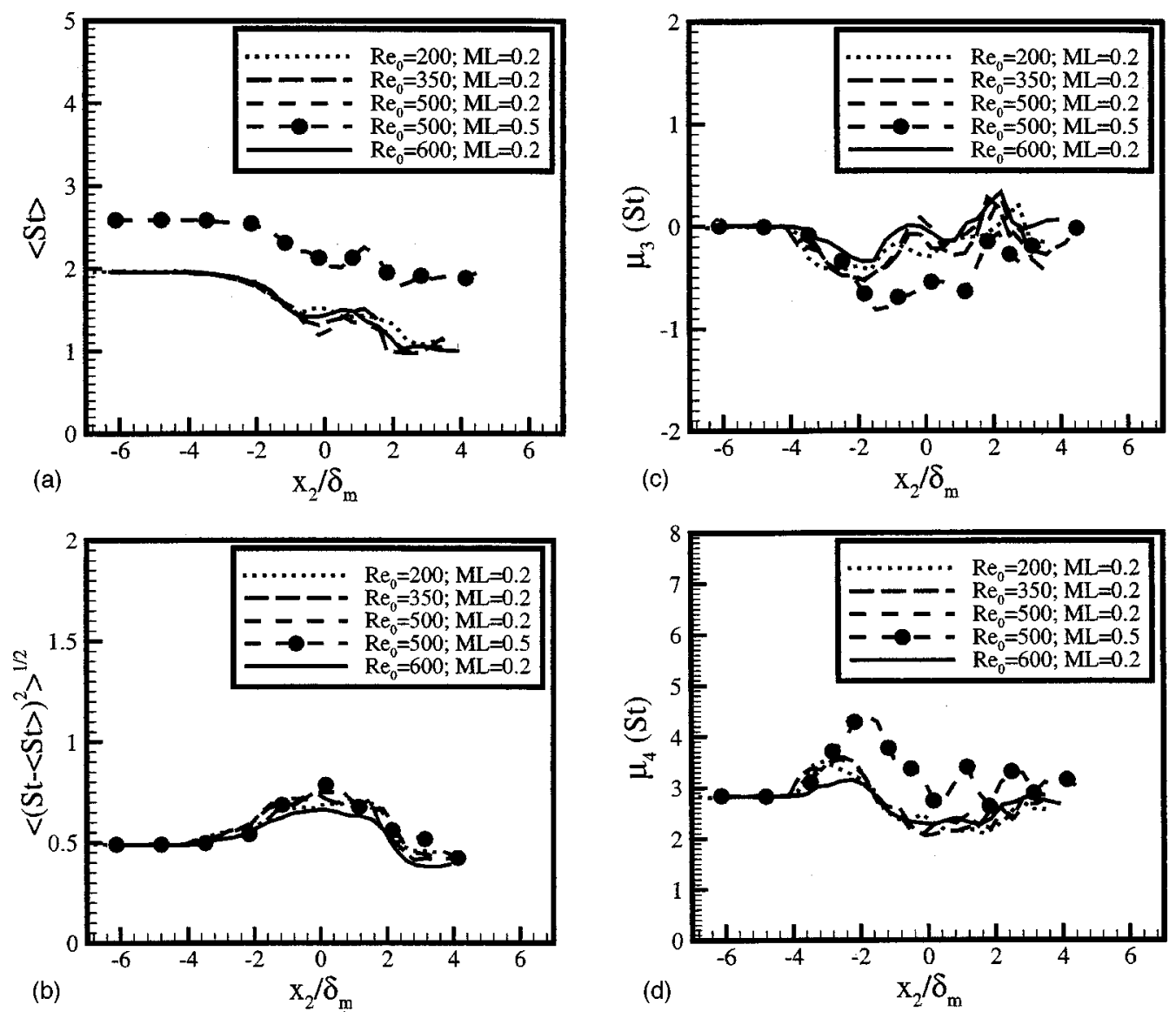

FIG. 7. Droplet Stokes number ( $\mathrm{St}=\tau_{d} \Delta U_{0} / \delta_{\omega, 0}$ ) planar-averaged statistics for various simulations at time $t \Delta U_{0} / \omega_{\omega, 0}=85$. (a) mean, (b) root mean square fluctuation, (c) skewness factor, and (c) flatness factor.

number statistics are almost uniform and a near-Gaussian $\mathrm{PDF}$ is retained for the laden stream, $x_{2} / \delta_{m}<-4$. At the laminar-turbulent boundary of the lower stream near $x_{2} / \delta_{m} \approx-2$, droplets from both the interior turbulent fluid and exterior laminar fluid regions are being sampled in the statistics. This results in increased intermittency and deviations from Gaussian behavior; particularly for the $\mathrm{ML}=0.5$ case, which saturates earlier. However, for the interior portion of the mixing layer, $x_{2} / \delta_{m}>-1$, more uniform distributions of droplets are sampled (i.e., from purely turbulent fluid) and closer to Gaussian statistics are recovered. These results regarding the droplet size distributions and the effect of $\mathrm{ML}$ are in good qualitative agreement with the earlier finding in MBI, despite the monodisperse initialization used in the former study. Figure 7 also indicates that the final time state of the mixing layers remains that of a two-phase flow with significant droplet mass in the laden stream. At time $t \Delta U_{0} / \delta_{\omega, 0}=85$ the relative mass of liquid to gas in the $x_{2} / \delta_{m}<-4$ portion of the layers is approximately $11 \%$ and $41 \%$ for the $\mathrm{ML}=0.2$ and $\mathrm{ML}=0.5$ flows, respectively. Throughout the remainder of the paper we will be focusing primarily on these final time states of simulations TP500b and TP600.

\section{B. Subgrid analysis}

As mentioned above, large eddy simulation (LES) involves performing a filtering operation on the governing equations in order to effectively remove the smallest, unresolvable scales of the flow under investigation. ${ }^{12,13}$ The missing information results in "Reynolds stress like" terms that then require modeling to close the system of the LES equations. In the context of the present two-phase flow, this corresponds to filtering the governing equations (1)-(5). Each droplet will "feel" effects from both the resolved and unresolved subgrid Eulerian flow variables. This statement translates mathematically in Eqs. (6) -(9) through the potential influence of all subgrid variables on the droplet evolution. For example, if we denote the filtered velocity field by $\overline{u_{i}}$ (defined below), then the subgrid velocity fluctuation can be defined as $u_{i}^{\prime}=u_{i}-\bar{u}_{i}$ and the instantaneous velocity appearing in the droplet drag force is substituted with the sum of the resolved and unresolved velocities; $u_{i} \rightarrow \overline{u_{i}}+u_{i}^{\prime}$. Although a variety of past research exists that studies solid particle transport through turbulent flow fields generated by LES, ${ }^{14-19}$ the vast majorities of the investigations completely neglect the influence of the subgrid velocity $u_{i}^{\prime}$ on the particle trajectories. This neglect is done under the a priori, and untested, assumption that these effects will be negligible due to the particle's finite inertia. That is, it is assumed that heavy particles are not "jostled" significantly by the smallest eddies due to their inertia, and hence only the resolved velocity field needs to be considered (despite the fact that particles with $\mathrm{St} \sim 1$ are generally considered). Note that the above cited LES aim at predicting the "true" particle trajec- 
tories through the flow field, not a filtered droplet velocity field (filtering a Lagrangian point particle is meaningless in the context of LES). As mentioned above, for evaporating droplets all thermodynamic flow variables appear in the droplet transport equations $\left(\rho, T, P\right.$, and $\left.Y_{V}\right)$ along with their corresponding subgrid fluctuations, which complicates the matter even further. However, if the effects of $u_{i}^{\prime}$ are truly negligible, it seems reasonable to neglect all other subgrid quantities using the same argument, since the droplet velocity, temperature, and mass equations all have exponential decay time scales approximately equal to $\tau_{d}$ [see (6)-(9)].

The DNS presented in this paper are ideal for evaluating the effect of subgrid fluctuations on the droplet transport due to their large resolutions, number of droplets, and relatively large Reynolds numbers. We will not address the filtered Eulerian gas phase equations, nor their modeling in this paper. Several detailed investigations of this kind have already appeared in the literature in which the behavior of various subgrid models are investigated using both $a$ priori and $a$ posteriori analyses of compressible mixing layers. ${ }^{30,31} \mathrm{Al}-$ though these studies do not explicitly address two-phase flows, the LES modeled Navier-Stokes equations can be used unchanged in their single-phase form to predict oneway coupled particle laden flows. On the other hand, gas phase subgrid models and methods for treating the phase coupling terms for two-way coupled LES remain unresolved issues; however, these are not the subject of the present paper but should receive future attention.

We begin by presenting some preliminary definitions: The filtered quantity $\bar{\phi}$ is related to the instantaneous field $\phi$ through the physical space filter defined by

$$
\bar{\phi}=\int \phi\left(y_{i}\right) G_{\Delta}\left(x_{i}-y_{i}\right) d^{3} y_{i},
$$

where $G_{\Delta}$ is the filter function with the requirement that $\int G_{\Delta}\left(y_{i}\right) d^{3} y_{i}=1$. The flows of interest exhibit variable gas density, even for low Mach numbers due to droplet evaporation. It is therefore convenient to define Favre density weighted filtered variables as $\widetilde{\phi}=\overline{\rho \phi} / \bar{\rho}$. The fluctuations with respect to both the non-weighted and weighted filtered variables are $\phi^{\prime}=\phi-\bar{\phi}$ and $\phi^{\prime \prime}=\phi-\widetilde{\phi}$, respectively. For variable density flows of the type considered in this study, the velocity, temperature, and vapor mass fraction appear most naturally in the Favre filtered form in the LES equations, whereas the density and pressure appear most naturally as non-weighted filtered variables (e.g., Refs. 30 and 31). Therefore, hereinafter, all filtered and subgrid analyses will be conducted using $\bar{\rho}, \widetilde{u}_{i}, \widetilde{T}, \bar{P}$, and $\widetilde{Y}_{V}$ for the resolved field, and $\rho^{\prime}, u_{i}^{\prime \prime}, T^{\prime \prime}, P^{\prime}$, and $Y_{V}^{\prime \prime}$ for the subgrid. These will be assumed to be the actual fields that are available and/or require modeling for the droplet transport in LES (i.e., we do not analyze $\overline{u_{i}}$ or $u_{i}^{\prime}$ since these will not appear in the LES equations).

A wide variety of filter functions have been considered in the LES literature including both physical space filters and spectral space filters (e.g., Ref. 32). In the present paper only physical space filters are discussed, as spectrally filtered fields do not satisfy the realizability condition. The two filters of interest are the "cubic top-hat" filter:

$$
G_{\Delta}^{\mathrm{CTH}}\left(x_{i}\right)=\frac{1}{\Delta_{f}^{3}} \prod_{\alpha=1}^{3}\left[H\left(\Delta_{f} / 2-\left|x_{\alpha}\right|\right)\right],
$$

and the "spherical top-hat" filter:

$$
G_{\Delta}^{\mathrm{STH}}\left(x_{i}\right)=\frac{6}{\pi \Delta_{f}^{3}} H\left(\Delta_{f} / 2-\left|x_{i}\right|\right),
$$

where $H(x)$ is the Heaviside function and $\Delta_{f}$ is the filter width. The cubic top-hat filter is the simplest to integrate in actual applications yet suffers from the fact that transport equations filtered using this function are no longer invariant to coordinate rotations due to the anisotropy of the kernel function. ${ }^{32}$ In contrast, the spherical top hat is isotropic and, therefore, the rotational symmetry of the governing equations is preserved after filtering. Although this preservation is important from a theoretical perspective, the majority of LES studies do not consider this effect and work with anisotropic box filters. A Gaussian spatial filter also appears in the literature and has the further advantage of being positive definite in both physical and wave number space (the top hats are oscillatory in wave number space); however, it requires integration over the entire domain and is therefore the least computationally efficient. Therefore, Gaussian filters are not considered in this paper.

\section{Filtered DNS}

The two filters described above are applied in an a priori manner to the DNS database in order to analyze the subgrid fluctuation fields in the context of two-phase turbulent flows. Since LES is meant to be conducted for relatively large Reynolds number flows, it is important to have a DNS subgrid that is well developed and permits the performance of subgrid analyses. Although detailed subgrid information is almost exclusively extracted from DNS generated flow fields, experimental data [from two-dimensional (2D) planes] have also been used successfully in single-phase evaluations; ${ }^{33,34}$ however, measurements of the type required in the present two-phase study are beyond the current capabilities of experiments. For droplet laden flows, the simulations described in Table II are ideally suited to this task, due to both the large resolutions and number of droplets. This allows us to use larger filter widths than are available in standard, lower resolution, DNS studies, and to therefore obtain more robust subgrid statistics. For example, the largest filter width that will be used below is $\Delta_{f} \approx \delta_{\omega, 0}$. For simulation TP600 this corresponds to $\Delta_{f}=11 \Delta x$; leading to averaging volumes that contain $11^{3}=1331$ grid points for the cubic box filter, and $\pi 11^{3} / 6=697$ grid points for the spherical top-hat filter. As a comparison, recent single-phase LES studies of the temporal mixing layer have considered cubic filters having widths as large as $\Delta_{f}=2.2 \Delta x$ (Ref. 35) and $\Delta_{f}=1.8 \Delta x$ (Ref. 31). Finally, case TP600 and TP500b are initialized with an average of 367 and 697 droplets within the cubic filter with $\Delta_{f}$ $\approx \delta_{\omega, 0}$ (see Table II), respectively, and therefore provide very reasonable droplet statistics. 

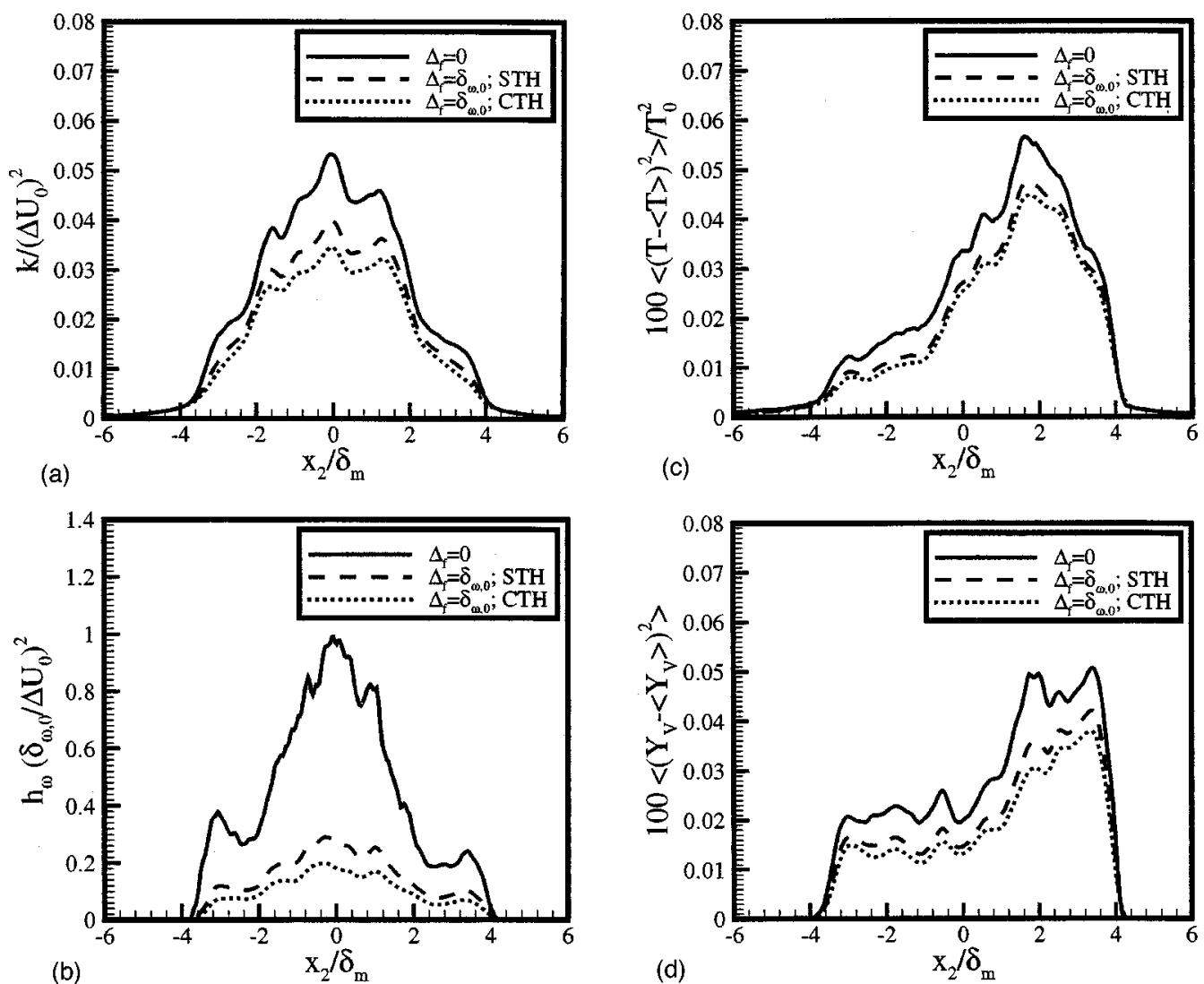

FIG. 8. Planar-averaged statistics for various simulations at time $t \Delta U_{0} / \delta_{\omega, 0}=85$ calculated based on both unfiltered and filtered gas phase fields. (a) fluctuation kinetic energy, (b) fluctuation enstrophy, (c) temperature fluctuation variance, and (d) vapor mass fraction fluctuation variance.

In contrast to analyzing the data using only the "coarse mesh" (i.e., only those grid points available on the hypothetical LES mesh), all analyses are performed on the fine (DNS) mesh using all grid points. Distinguishing between the two meshes becomes important when particular types of analyses are performed, such as when derivatives of the variables are calculated; however, the distinction will not be important for the following analyses. Nevertheless, the choice of $\Delta_{f} \approx \delta_{\omega, 0}$ corresponds to reasonable hypothetical coarse mesh LES resolutions for run TP600, which range from 27 $\times 30 \times 16$ to $54 \times 60 \times 32$ grid points for relative filter widths of $1 \leqslant \Delta_{f} / \Delta x^{(\mathrm{LES})} \leqslant 2$, where $\Delta x^{(\mathrm{LES})}$ is the LES mesh spacing. Note that $\Delta x^{(\text {LES })}$ does not have to equal the filter width applied to the governing equations, and the ratio $\Delta_{f} / \Delta x^{(\mathrm{LES})}=2$ is often recommended due to the smoother fields being resolved on the coarse mesh (e.g., Ref. 30). Hereinafter, we will only analyze results from the fully developed $\left(t \Delta U_{0} / \delta_{\omega, 0}=85\right)$ flow fields from the two largest simulations, TP600 and TP500b, due to their fine resolutions, relatively large Reynolds number, and large numbers of droplets; however, many of the trends discussed have been checked against results from the remaining cases. Additionally, all filter widths are chosen to be odd integer values of the DNS grid spacing so that the "LES nodes" directly overlap the fine mesh nodes.

As an example of the relative small-scale energy in the flow fields both before and after filtering, consider Fig. 8, which depicts planar-averaged fluctuation intensities based on both the DNS and filtered DNS data as a function of $x_{2}$ for run TP600. The mean turbulence kinetic energy $(k)$ and enstrophy $\left(h_{\omega}\right)$ are defined in the standard manner:

$k=\frac{1}{2} \sum_{\alpha=1}^{3}\left\langle\left(u_{\alpha}-\left\langle u_{\alpha}\right\rangle\right)^{2}\right\rangle, \quad h_{\omega}=\sum_{\alpha=1}^{3}\left\langle\left(\omega_{\alpha}-\left\langle\omega_{\alpha}\right\rangle\right)^{2}\right\rangle$.

The three curves in each of the figures correspond to the averages based on the instantaneous DNS fields, as well as on fields produced by filtering the DNS flows using both the spherical top hat (STH) and cubic top hat (CTH) filters (e.g., $u_{i} \rightarrow \widetilde{u}_{i}$ in calculating $k$ ). The extent of separation between these curves in each of the figures provides a direct measure of the relative energy in each of the subgrids. By far, the largest amount of small-scale subgrid energy is found in the vorticity field, since differentiating the velocity obviously amplifies the small-scale contribution of the spectrum; filtering removes a large amount of this energy [Fig. 8(b)]. The kinetic energy [Fig. 8(a)] also exhibits a relatively large loss of energy through filtering. On the other hand, the temperature [Fig. 8(c)] and vapor mass fraction [Fig. 8(d)] variances show the smallest effects from filtering. This is to be expected since the Prandtl number is $\operatorname{Pr}_{G}=0.697$ (Table I) and the Lewis number is equal to unity; therefore, the smallest scales (the Batchelor scales) for these scalar variables are larger than the Kolmogorov scale of the turbulence and less energy is truncated by the filtering operation. Mean velocity, temperature, and mass fraction profiles have also been exam- 

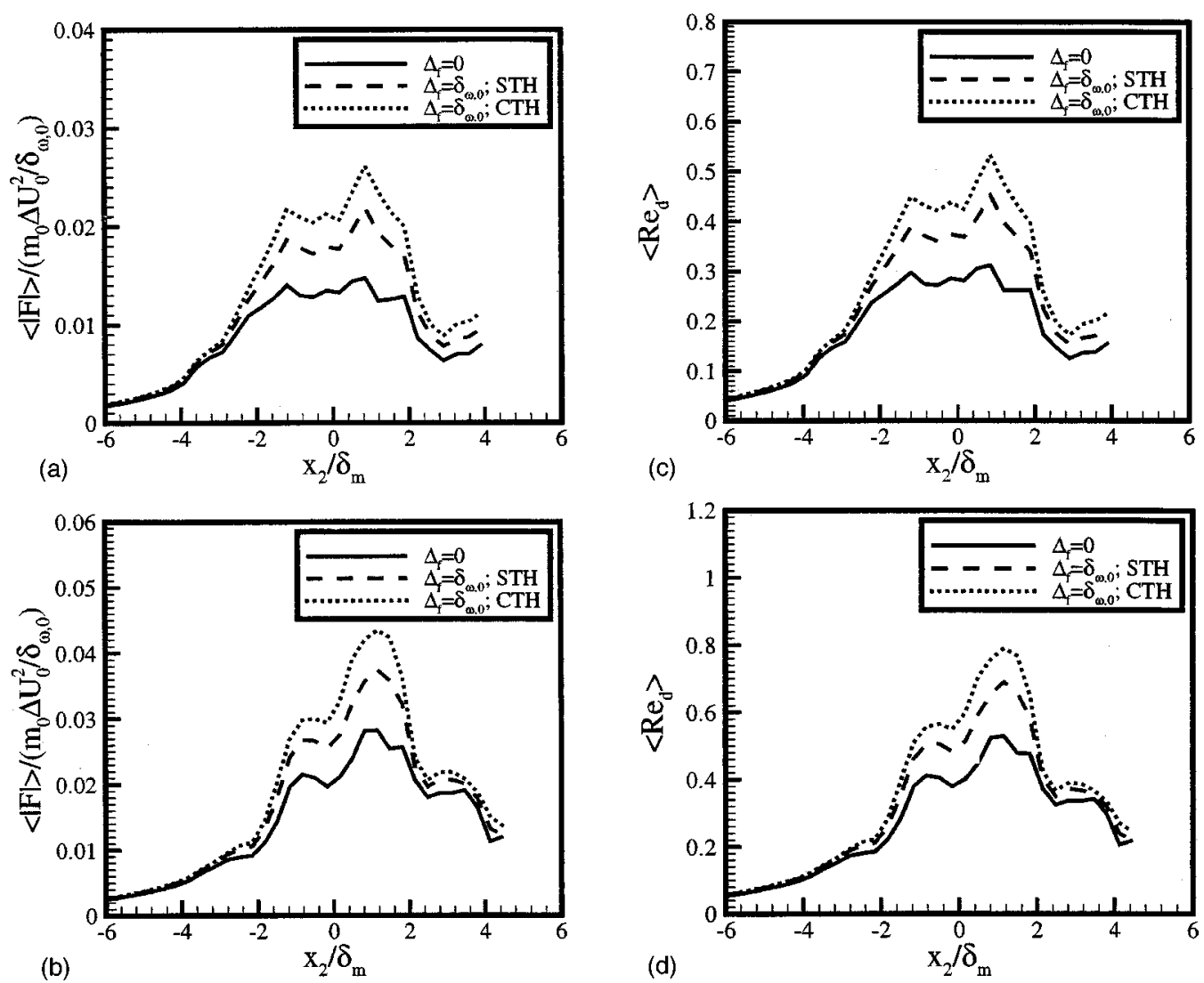

FIG. 9. Planar-averaged statistics at droplet locations for various simulations at time $t \Delta U_{0} / \delta_{\omega, 0}=85$ calculated based on both unfiltered and filtered gas phase fields. (a) Droplet drag force for simulation TP600, (b) droplet drag force for simulation TP500b, (c) droplet Reynolds number for simulation TP600, and (d) droplet Reynolds number for simulation TP500b. Note that $m_{0}$ is a reference value corresponding to the mass of a droplet with $\mathrm{St}=\langle\mathrm{St}\rangle_{0}=3$.

ined in a similar manner. Although the unfiltered and filtered profiles are not identical, no significant visible separations of the curves are observed (not shown). Finally, Fig. 8 reveals that there can be a significant difference between the energy truncated by the cubic and spherical filter kernels for fields characterized by relatively extensive small-scale energy; with obviously greater truncation for the larger volume cubic filter.

\section{Subgrid effects on droplet transport}

We now turn to the Eulerian subgrid effects on the transport and vaporization of the droplets. In order to gauge these effects we perform an analysis similar to that in Fig. 8. However, we now compute planar averages of droplet transport variables as functions of both the unfiltered (DNS) and the filtered Eulerian variables interpolated to the droplet positions. Figure 9 shows both the averaged droplet drag force magnitude [Eq. (7)] and droplet Reynolds number calculated in this manner from simulations TP600 and TP500b $\left[m_{0}\right.$ in Figs. 9(a) and 9(b) is a reference value corresponding to the mass of a droplet with $\mathrm{St}=\langle\mathrm{St}\rangle_{0}=3$ ]. Clearly, a substantial change is produced in both the drag force [Figs. 9(a) and 9(b)] and the droplet Reynolds numbers [Figs. 9(c) and 9(d)] by the filtering operation. This is direct evidence that errors will be produced by performing a LES that explicitly neglects the influence of subgrid fluctuations $\left(\rho^{\prime}\right.$ and $\left.u_{i}^{\prime \prime}\right)$ on the droplet and/or solid particle transport. There is, however, a somewhat peculiar feature to the results in Fig. 9, in that the drag force (and $\mathrm{Re}_{d}$ ) magnitudes are increased when they are based on the filtered Eulerian fields, despite the fact that energy has been removed from the velocity field. To understand why this occurs, consider a particle with $\mathrm{St} \ll 1$ convected by the DNS flow. In this case the instantaneous slip velocity (and drag force) will be nearly negligible at any given particle position. If the Eulerian velocity is then filtered, the recalculated slip velocity will now be much larger due to the fact that $u_{i}$ is replaced by $\widetilde{u}_{i}$, while $\mathrm{v}_{i}$ remains the same. This indicates that the increasing drag forces observed in Fig. 9 are somewhat biased: In a real LES calculation, the particles will adjust to the filtered flow as they evolve in time and the mean drag forces, which are primarily governed by $\tau_{d}$, will be much closer to the values calculated from the DNS flow in Fig. 9. This means that the proper interpretation of Fig. 9 is that in a LES in which the subgrid is neglected, there may be a substantial error in the prediction of the "true" particle velocities; i.e., $\mathrm{v}_{i}\left(\widetilde{u}_{i}\right) \neq \mathrm{v}_{i}\left(u_{i}\right)$. Figure 9 also shows that this error can be substantial, even for both St $\sim 1$ and $\operatorname{Re}_{d} \sim 1$. Finally, Figs. 10(a) and 10(b) depict the corresponding averaged values of the droplet heat flux, $Q$ [see Eq. (8)], and the nondimensional evaporation rate, $\beta$, from simulation TP600. Similar to the above discussions, both of these quantities are observed to be significantly affected by the Eulerian subgrid (though to a lesser extent due to the Prandtl number). 

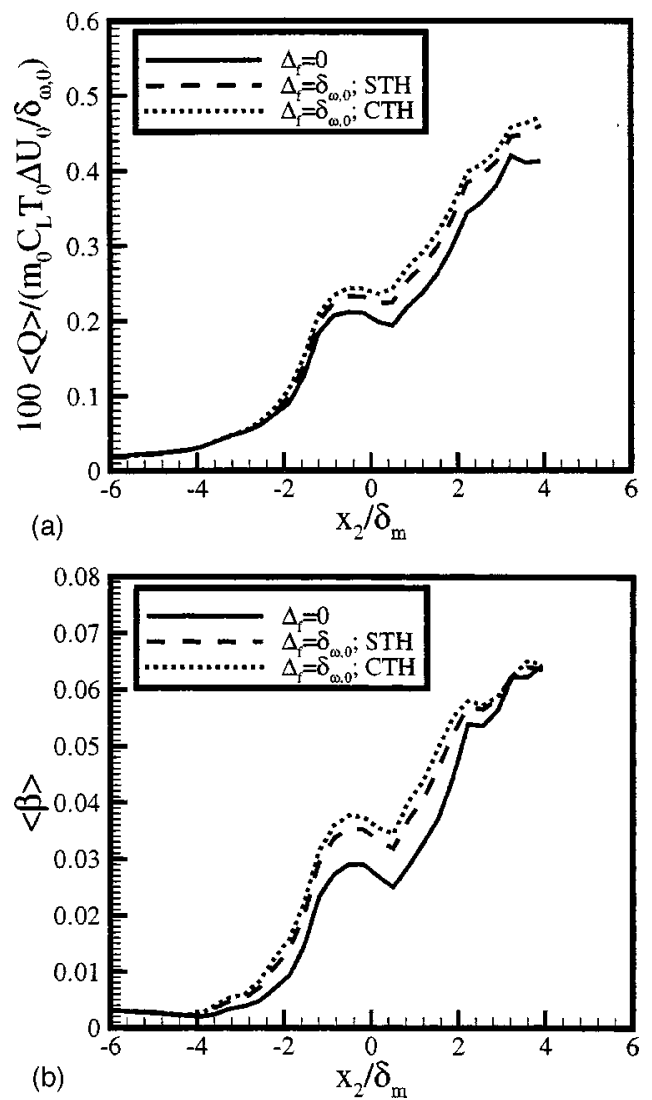

FIG. 10. Planar-averaged statistics at droplet locations for simulation TP600 at time $t \Delta U_{0} / \delta_{\omega, 0}=85$ calculated based on both unfiltered and filtered gas phase fields. (a) Droplet heat flux, and (b) a droplet nondimensional evaporation parameter.

A further indication of the influence of the subgrid on the droplet trajectories is provided by considering relative time scales. We define a "subgrid Stokes number:' $\mathrm{St}_{\mathrm{sg}}$ $=\tau_{d} / \tau_{\mathrm{sg}}$, where the subgrid time scale is based on the subgrid velocity variance and the filter width, $\tau_{\mathrm{sg}}=\Delta_{f} /{\widetilde{u^{\prime \prime}} u^{\prime \prime}}^{1 / 2}$. In relation to the previously defined Stokes number $(\mathrm{St})$ :

$$
\frac{\mathrm{St}_{\mathrm{sg}}}{\mathrm{St}}=\frac{\widetilde{u^{\prime \prime} u^{\prime \prime}}{ }^{1 / 2} / \Delta U_{0}}{\Delta_{f} / \delta_{\omega, 0}} .
$$

Subgrid effects on the droplet trajectories can only be neglected safely for a subgrid Stokes number substantially greater than unity. Since the normalized subgrid velocity in the numerator is typically much less than unity, the subgrid Stokes number will only exceed the "'macro-' Stokes number for very small filter widths. For example, an examination of the present results [see Fig. 5(a)] shows that $\mathrm{St}_{\mathrm{sg}} \sim 10^{-1}$, which indicates that the droplets will closely follow the subgrid fluctuations for the present conditions.

\section{E. Subgrid modeling}

In this section we propose an extension of the "eddy interaction model'" (EIM) as a means of modeling the missing subgrid fluctuations that appear in the LES droplet transport equations. The EIM ${ }^{36}$ is the most commonly used model in multiphase Reynolds averaged simulations. In RANS calculations only the ensemble- (or time-) averaged velocity field is known, and the effects of the entire "subgrid" velocity fluctuations - the rms velocity-on the particle trajectories must be modeled. In the EIM the rms velocity is calculated by sampling from a Gaussian PDF using a random number generator. The PDF has zero mean and a variance usually obtained by a transport equation for the turbulence kinetic energy; as is already available in a $k-\epsilon$ model. These values are then added to the mean velocity values at the particle locations, producing an isotropic turbulence. Closure of the EIM then requires only a model for the particle's " "eddy interaction time," which is equivalent to determining how many time steps this sampled rms velocity is used before a new random value is chosen. In its simplest form, the EIM is employed by sampling new velocities at each time step (white noise in time); however, many alternative, and increasingly complex, versions have been proposed that incorporate anisotropy and models for the Lagrangian time correlation matrix in order to obtain more accurate residence times (e.g., Refs. 36 and 37).

We propose extending the EIM model to account for the effects of not only the subgrid velocity, but also of all of the Eulerian subgrid fluctuations that appear in the droplet transport equations $\left(\rho^{\prime}, u_{i}^{\prime \prime}, T^{\prime \prime}, P^{\prime}\right.$, and $\left.Y_{V}^{\prime \prime}\right)$ [note that the pressure variance is needed to evaluate the vapor surface mass fraction (11)]. In the context of LES, the effects of the subgrid velocity fluctuations on the particle dynamics are much less significant than in RANS, where all of the turbulence energy needs to be modeled. It may therefore be reasonable to expect that the extended EIM need not be as complex, as its RANS versions in order to obtain satisfactory droplet transport statistics. Three issues are involved in extending the EIM: (1) determine the eddy interaction time appropriate for LES; (2) determine the form of the subgrid PDFs; and (3) estimate the subgrid fluctuation variances. The first issue is the easiest to resolve since in LES the appropriate length scale for the "subgrid eddies" is $\Delta_{f}$. This leads to an interaction time equal to $\Delta_{f} /\left|v_{i}\right|$ as a first approximation. Estimating the form of the subgrid PDFs is not as simple due to intermittency effects that can lead to strong deviations from Gaussian behavior in the small scales of turbulence (discussed below). Additional uncertainties are associated with choosing a form for the vapor mass fraction subgrid PDF, as will also be shown below. Finally, the third issue requires modeling the subgrid variances for each of the Eulerian variables at the droplet locations. Of course, transport equations for each of the variances could be derived and modeled just as the kinetic energy equation is used in RANS. However, this would introduce undesirable complexity to the LES equations. In what follows, we will propose using a scale similarity model to obtain the variances directly from the resolved field information.

\section{Subgrid probability density functions}

Before considering how to model the missing subgrid information needed for the droplet transport in LES, it is useful to briefly review what is already known about turbulent distributions. Turbulent velocity fields are generally considered to be well modeled by the Gaussian PDF that is used in the standard EIM (though there is recent evidence to the 
contrary) $;^{38}$ however, in LES it is the small subgrid scales that are of interest. It is well known that the small scales of the dissipation range are highly intermittent, as evidenced by larger than Gaussian, or exponential, tails in the PDFs of velocity differences and derivatives. ${ }^{39}$ On the other hand, passive scalar distributions are highly dependent on initial conditions, even at large scales. Scalars are generally modeled by bounded PDFs such as the amplitude mapping closure, logit-normal, or $\beta$ frequencies that are capable of predicting an entire range of PDF shapes from initially segregated species (in the case of mass fractions) to asymptotically Gaussian. ${ }^{40}$ Turbulently advected scalars are also characterized by highly intermittent small scales, ${ }^{41}$ and even the "long time" PDFs of the scalar itself can show substantial deviations from Gaussian behavior. ${ }^{42}$ Only relatively few studies have investigated the form of subgrid PDFs in turbulent flows and these are generally limited to the mixture fraction field in the context of combustion modeling. Madnia and $\mathrm{Givi}^{43}$ first proposed closing the subgrid (constant rate) reaction source term by assuming a $\beta$ PDF for the subgrid mixture fraction distribution. The $\beta \mathrm{PDF}$ is then specified based on the subgrid mean and variance that determine the two parameters of the model. Cook and Riley ${ }^{44}$ proposed calculating the subgrid variance directly from the resolved scalar field using a scale similarity model. Comparisons with DNS generated scalar fields showed that integrated mean product concentrations are reasonably well predicted using this approach, despite finding that the actual subgrid PDFs are not well predicted by the $\beta$ form (see also Refs. 35, 45, and 46).

In extending the EIM for the presently required subgrid modeling, we adopt a similar procedure as that described above for scalar fields; i.e., we assume a form for the subgrid PDF. However, instead of assuming a form for the entire distribution we will only consider modeling the actual zero mean fluctuations. In this case, each droplet will "feel" the effects from both a deterministic filtered mean gas phase variable (known on the coarse mesh) plus a second superimposed contribution from the corresponding fluctuation represented by a zero mean stochastic variable. This latter fluctuation will be assumed to have a particular PDF that remains to be modeled and that can be obtained from knowing only the subgrid variance (for "two-parameter" PDFs). The distinction is purely conceptual for distributions such as the Gaussian, for which the mean can be subtracted without changing the form of the PDF. However, if a particular scalar field is well represented by the $\beta \mathrm{PDF}$, then its zero mean fluctuation can no longer be assumed to also be $\beta$ distributed. Note also that the subgrid PDF only refers to the theoretical distribution of the fluctuations within each subgrid volume used in the filtering operation. ${ }^{35,43}$ Our approach therefore contains the implicit assumption that every point that has the same subgrid variance for any given Eulerian variable (as the droplet locations) also has the same theoretical fluctuation distribution.

Before proceeding to analyze the actual subgrid PDFs, we first examine the globally calculated PDFs of the subgrid fluctuations. In the following analyses we will consider only the cubic top-hat filter due to the larger relative subgrid contributions available using this kernel function. Several ex-
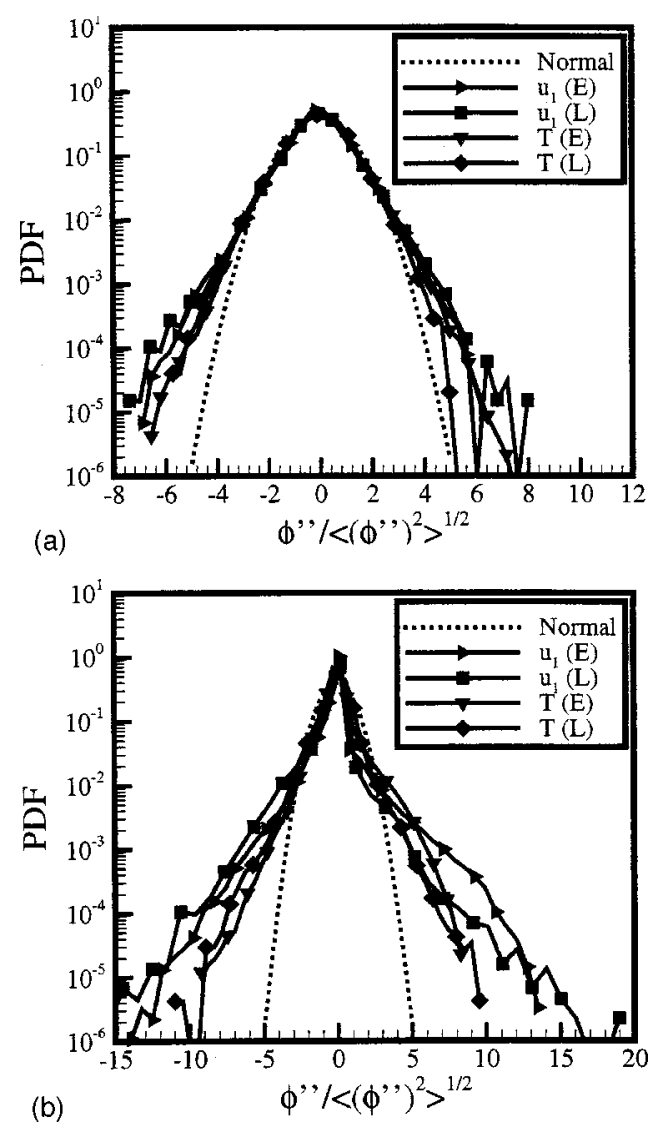

FIG. 11. Normalized PDFs of Favre subgrid fluctuations calculated for $x_{1}$ $-x_{3}$ planes from simulation TP600 at time $t \Delta U_{0} / \delta_{\omega, 0}=85$ with $\Delta_{f}$ $=\delta_{\omega, 0}$. (a) $x_{2}=0 \pm \delta_{m} / 2$, and (b) $x_{2}=-3.5 \delta_{m} \pm \delta_{m} / 2$.

amples of these PDFs calculated from simulation TP600 at time $t \Delta U_{0} / \delta_{w, 0}=85$ are presented in Fig. 11. The curves correspond to the measured distributions of both the streamwise velocity and temperature Favre fluctuations measured at two different $x_{2}$ locations; at the centerline for Fig. 11(a) and along the laden stream turbulent-laminar fluid boundary at $x_{2} / \delta_{m}=-3.5$ (in both cases the sampling bin has a thickness equal to $\left.\pm \delta_{m} / 2\right)$. The designations (E) and (L) in the figure captions denote PDFs calculated based on all Eulerian grid points, and those calculated at all Lagrangian droplet locations, respectively. Both the velocity and the temperature are characterized by PDFs with significantly larger than Gaussian tails (the dotted curve is the zero mean, unity variance Gaussian). These results clearly indicate that the small-scale fluctuations display relatively strong intermittency effects, with departures from Gaussian PDFs that are markedly enhanced along the mixing layer boundary. Although the PDFs presented in this manner do not directly correspond to the subgrid PDFs we wish to model, these results, nevertheless show that it cannot simply be assumed that a subgrid EIM can be based on the same Gaussian statistics that are used in RANS models. This is further evidenced in Fig. 12, which shows the cross stream-dependent skewness and flatness factors for the three velocity components calculated at the Eulerian grid points from the same TP600 flow field. The subgrid fluctuations for all of the components show strong deviations from Gaussian behavior (i.e., $\mu_{3}=0$ and $\mu_{4}=3$ ) 

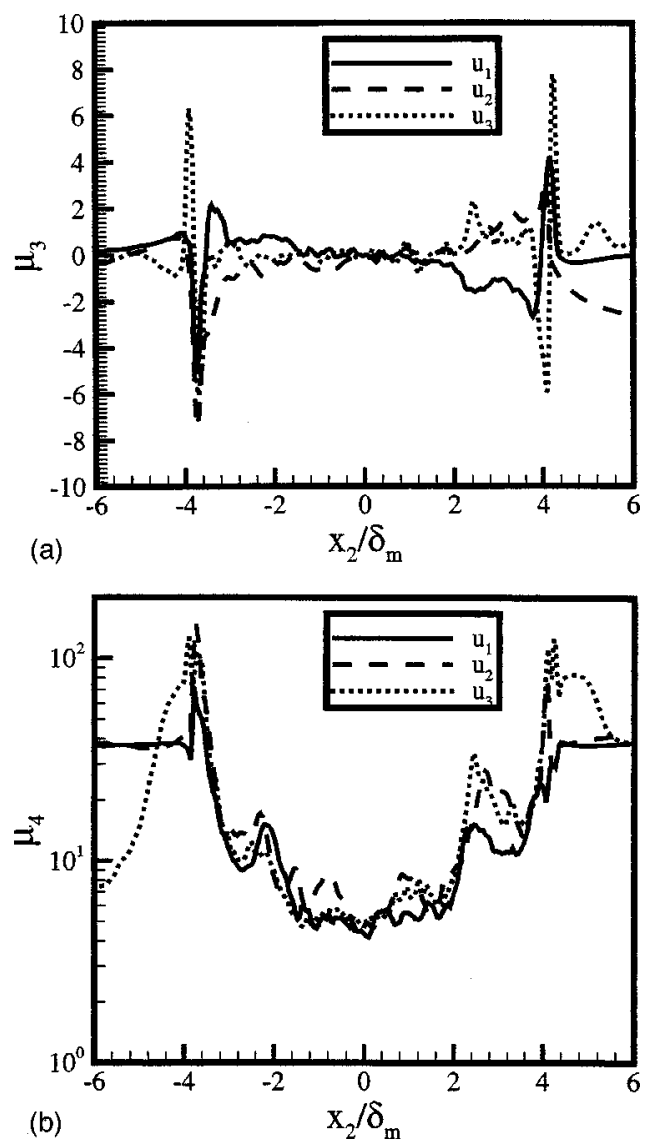

FIG. 12. Moments of velocity subgrid Favre fluctuations calculated at Eulerian grid points from simulation TP600 at time $t \Delta U_{0} / \delta_{\omega, 0}=85$ using $\Delta_{f}$ $=11 \Delta x \approx \delta_{\omega, 0}$.

at all $x_{2}$ locations. Notice that the intermittency effects are dramatically enhanced near both edges of the mixing layer due to the "cross sampling" of both turbulent and laminar fluid (see e.g. Fig. 4).

To illustrate the forms of the actual subgrid PDFs representing the form of distributions within $\Delta_{f}^{3}$ averaging volumes, we investigate the subgrid skewness and flatness factors. Cook and Riley ${ }^{44}$ pointed out that the subgrid variance must be calculated as $\overline{\phi^{\prime} \phi^{\prime}}=\overline{\phi^{2}}-\bar{\phi}^{2}$ since the subgrid mean $\bar{\phi}$ at the coarse mesh point represents the entire subgrid volume. In a consistent manner, we calculate the subgrid skewness $\left(\mu_{3}^{\mathrm{sg}}\right)$ and the subgrid flatness $\left(\mu_{4}^{\mathrm{sg}}\right)$ factors as

$$
\mu_{3}^{\mathrm{sg}}(\phi)=\frac{\overline{\phi^{3}}-3 \bar{\phi} \overline{\phi^{2}}+2 \bar{\phi}^{3}}{\left(\overline{\phi^{2}}-\bar{\phi}^{2}\right)^{3 / 2}},
$$

and

$$
\mu_{4}^{\mathrm{sg}}(\phi)=\frac{\overline{\phi^{4}}-4 \bar{\phi} \overline{\phi^{3}}+6 \bar{\phi}^{2} \overline{\phi^{2}}-3 \bar{\phi}^{4}}{\left(\overline{\phi^{2}}-\bar{\phi}^{2}\right)^{2}} .
$$

Favre filtered variables are substituted into the above expressions if $\phi$ is either $u_{i}, T$, or $Y_{V}$; i.e., $\bar{\phi} \rightarrow \widetilde{\phi}$. Rather than examining individual subgrid moments measured at particular locations, we look at planar averages of the moments (the averaging is performed after the local subgrid moments are calculated). These are presented in Fig. 13 for the fully developed TP600 flow averaged over both the Eulerian grid points and over moment values interpolated to the droplet locations. For all variables other than the vapor mass fraction, the actual subgrid distributions are observed to be much closer to Gaussian than the planar PDFs in Figs. 11 and 12. Intermittency effects are still found near the edges of the mixing layer; however, Gaussian statistics appear to be a reasonable approximation over much of the layer for these variables. Subgrid PDFs are sampled over relatively narrow spatial scales relative to the planar ensemble PDFs and therefore sample more homogeneous ensembles, thereby resulting in closer to Gaussian behavior. On the other hand, the vapor mass fraction PDFs are characterized by much larger deviations from Gaussian subgrid distributions, particularly near the edges of the mixing layer. Of course, the distributions in Fig. 13 may be more robustly modeled using an exponential PDF as a more general form than Gaussian; however, this would introduce an additional unknown (the exponent) into the model for which no closure is available.

It is interesting to note that the mass fraction PDFs in Fig. 13 show maximal flatness factors on opposite sides of the mixing layer for the Eulerian and droplet location interpolations. This occurs because the droplets tend to be found in regions of maximal vapor fraction; ${ }^{10}$ therefore the Eulerian and Lagrangian samplings can produce substantially different behavior. Figure 13 also suggests that the scalar fluctuation is not particularly well modeled by the Gaussian distribution over significant portions of the mixing layer; however, the adequacy of the Gaussian should be analyzed through future $a$ posteriori testing. Note, that the assumed $\beta$ PDF mentioned above applies only to the mixture fraction, and not to the individual species mass fractions being considered in this study.

\section{Subgrid variance prediction}

The last issue involved in extending the EIM is the determination of a model for the subgrid variances. Such a model should be relatively simple so as not to add dramatically to the complexity of the LES, and should also be applicable to all of the subgrid variances of interest; i.e., $\overline{\rho^{\prime} \rho^{\prime}}$, $\widetilde{u_{i}^{\prime \prime} u_{i}^{\prime \prime}}, \widetilde{T^{\prime \prime} T^{\prime \prime}}, \overline{P^{\prime} P^{\prime}}$ and $\widetilde{Y_{V}^{\prime \prime} Y_{V}^{\prime \prime}}$. Rather than adding additional transport equations for these terms into the LES formulation, we choose to model them based only on information available on the coarse LES mesh. A similarity model for the subgrid stress tensor proposed by Liu et al. ${ }^{33}$ is implemented for these purposes in the form

$$
\overline{\phi^{\prime} \phi^{\prime}}=C_{m}\left[\widehat{\bar{\phi}^{2}}-\hat{\tilde{\phi}}^{2}\right], \quad \overline{\phi^{\prime \prime} \phi^{\prime \prime}}=C_{m}\left[\widehat{\widetilde{\phi}^{2}}-\hat{\tilde{\phi}}^{2}\right],
$$

for both non-weighted and Favre variances. The right-hand side of each equation is calculated from the resolved field $(\bar{\phi}$ or $\widetilde{\phi}$ ) and $C_{m}$ is a model constant. The above model is calculated by applying a second "test filter" to the resolved fields having filter width $\widehat{\Delta_{f}} \geqslant \Delta_{f}$. No advantage is gained in using a Favre test filter; therefore, the filtering at level $\widehat{\Delta_{f}}$ is non-weighted for all variables. Cook and Riley ${ }^{44}$ also used this model to evaluate the subgrid scalar mixture fraction variance in LES of reacting turbulent flows. 

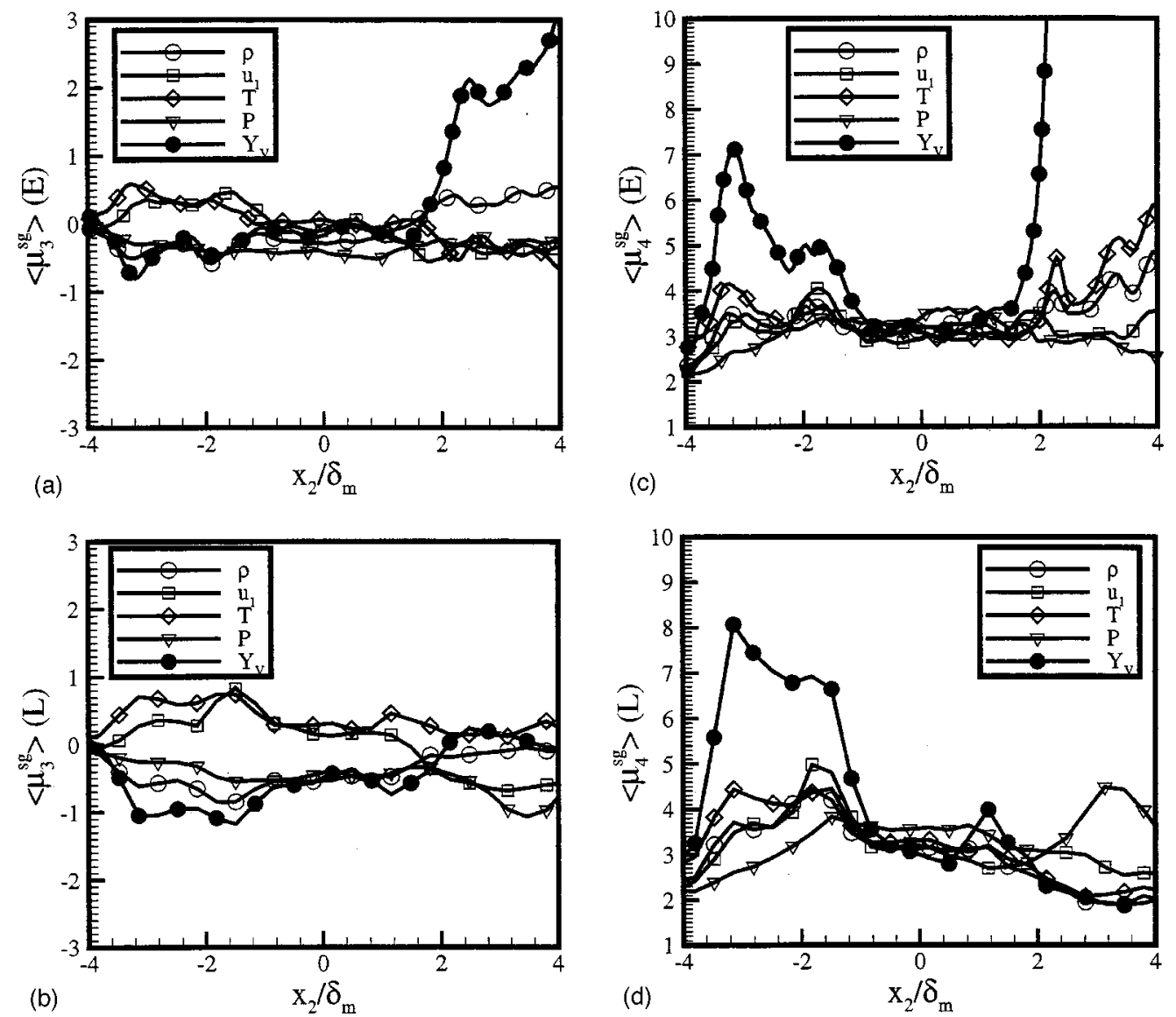

FIG. 13. Planar-averaged moments of subgrid PDFs calculated at time $t \Delta U_{0} / \delta_{\omega, 0}=85$ from simulation TP600 using $\Delta_{f}=11 \Delta x \approx \delta_{\omega, 0}$. (a) Skewness factor at Eulerian grid points, (b) skewness factor at droplet locations, (c) flatness factor at Eulerian grid points, and (d) flatness factor at droplet locations.

Before addressing the model constant $C_{m}$, we first examine the correlation between the model and the actual variance calculated from the DNS database. The correlation coefficient $(C)$, bounded by $-1 \leqslant C \leqslant+1$, is defined in the standard manner for any two variables, $\phi^{(1)}$ and $\phi^{(2)}$ :

$C\left(\phi^{(1)}, \phi^{(2)}\right)=\frac{\left\langle\left(\phi^{(1)}-\left\langle\phi^{(1)}\right\rangle\right)\left(\phi^{(2)}-\left\langle\phi^{(2)}\right\rangle\right)\right\rangle}{\left\langle\left(\phi^{(1)}-\left\langle\phi^{(1)}\right\rangle\right)^{2}\right\rangle^{1 / 2}\left\langle\left(\phi^{(2)}-\left\langle\phi^{(2)}\right\rangle\right)^{2}\right\rangle^{1 / 2}}$,

as a function of $x_{2}$, where the averaging is again over homogeneous $x_{1}-x_{3}$ planes for the mixing layer. Figure 14 shows the coefficient evaluated at time $t \Delta U_{0} / \delta_{\omega, 0}=85$ for simulations TP600 and TP500b. In this case, $\phi^{(1)}=\overline{\phi^{\prime} \phi^{\prime}}$ and $\phi^{(2)}=\widehat{\bar{\phi}^{2}}-\hat{\phi}^{2}$ in $(25)$. In calculating the correlations, it was necessary to reduce the original filter widths from those used previously to $\Delta_{f}=5 \Delta x$ (125 averaging points), in order retain a sufficient spacing between $\Delta_{f}$ and the test filter width $\widehat{\Delta_{f}}$. In Fig. 4, we have used $\widehat{\Delta_{f}}=11 \Delta x \approx \delta_{\omega, 0}$ and $\widehat{\Delta_{f}}$ $=9 \Delta x \approx \delta_{\omega, 0}\left(\widehat{\Delta_{f}} / \Delta_{f}=2.2\right.$ and $\left.\widehat{\Delta_{f}} / \Delta_{f}=1.8\right)$ in analyzing simulations TP600 and TP500b, respectively. A high degree of correlation is found between the model and the actual subgrid variances for both simulations and for all variables (the "drop" in the curve in Fig. 4(b) near $x_{2} / \delta_{m}=-3.6$ occurred because the subgrid variance fell below a lower tolerance level and the coefficient was set to zero). These results are consistent with additional tests that were performed using other filter widths and simulation results (not shown).

The model (24) has been investigated previously in the context of the subgrid Reynolds stresses by Liu et al. ${ }^{33}$ and also for modeling the mixture fraction variance by Cook and Riley ${ }^{44}$ and later by Jimenez et al. ${ }^{35}$ Liu et al. determined that the optimal model constant is $C_{m}=0.45 \pm 0.15$; however, when $C_{m}$ is calculated from an energy dissipation balance at the test grid level, a noticeable decreasing dependence on the filter width ratio, $\widehat{\Delta_{f}} / \Delta_{f}$, is observed (though still within the cited range). Cook and Riley also state that the constant is dependent on the filter ratio. They use $C_{m}$ $=0.5$ based on $\widehat{\Delta_{f}} / \Delta_{f}=1.8$. On the other hand, Jimenez et al. calculate the same constant analytically based on an assumed $-5 / 3$ spectral scaling and arrive at $C_{m}=1.69$ based on $\widehat{\Delta_{f}} / \Delta_{f}=2$ (and the smallest possible LES filter width; $\Delta_{f}=2 \Delta x$ ). They attribute the lack of agreement between their results and those of Cook and Riley on the fact that the Reynolds number of their DNS database ${ }^{25}$ is much larger. However, they do not mention the experimentally obtained model constant of Liu et al., which is also for moderately large Reynolds numbers; presumably because the subgrid stresses were measured as opposed to the scalar variance (though it is not clear why the distinction should make a significant difference for Schmidt numbers near unity; see 

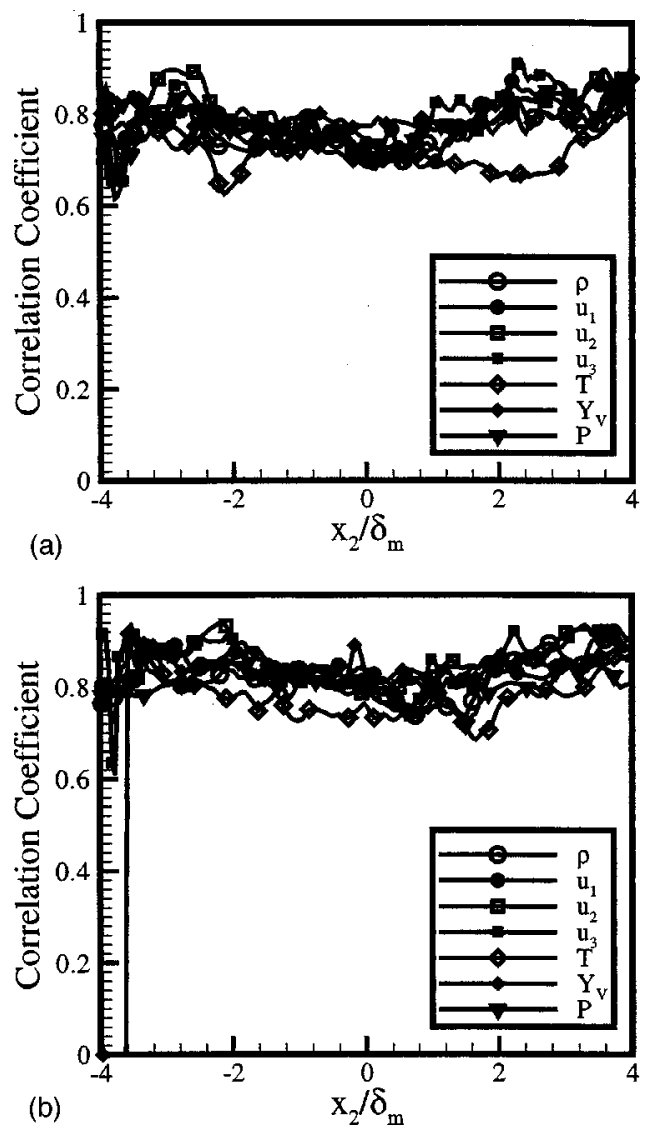

FIG. 14. Correlation coefficient for the exact and modeled subgrid variance calculated within $x_{1}-x_{3}$ planes at time $t \Delta U_{0} / \delta_{\omega, 0}=85$. (a) Simulation TP600 with $\Delta_{f}=5 \Delta x$ and $\hat{\Delta}_{f} / \Delta_{f}=2.2$, and (b) simulation TP500b with $\Delta_{f}=5 \Delta x$ and $\hat{\Delta}_{f} / \Delta_{f}=1.8$.

also below). As will be shown below, our own observations are more consistent with the measured model constant values of Liu et al. and Cook and Riley.

An analysis of the subgrids calculated from the present simulated two-phase flows reveals that the optimal model constant is a function of the filter width ratio, as discussed above. The constant $C_{m}$ is found to be well modeled using

$$
C_{m}=1+\frac{5}{12}\left(1-\widehat{\Delta}_{f} / \Delta_{f}\right) .
$$

This relatively simple form was obtained from a linear fit to both the TP600 results with $\widehat{\Delta_{f}} / \Delta_{f}=2.2$ and the requirement that $C_{m}=1$ for $\widehat{\Delta}_{f} / \Delta_{f}=1$ in order to recover the Bardina et al. ${ }^{47}$ model. The linear fit leads to $C_{m}=0.5$ for $\widehat{\Delta}_{f} / \Delta_{f}$ $=2.2$, and $C_{m}=0.67$ for $\widehat{\Delta}_{f} / \Delta_{f}=1.8$, which are the two filter ratios discussed in the preceding figures. Equation (26) will be shown below to work well for all variables of interest with the exception of the gas temperature for which consistent underpredictions of the subgrid variance were observed. This is probably due to a combination of latent heat effects and the larger length scales of the thermal field due to the Prandtl number effects (though the scalar field $Y_{V}$ does not show this feature even though the Lewis number is unity). In order to overcome this deficiency we use $C_{m} \rightarrow C_{m} / \operatorname{Pr}_{G}$ for the temperature subgrid variance predictions in what follows.

Planar-averaged predictions of the scale similarity model are presented in Fig. 15 for all of the variables needed in
LES of droplet laden turbulent flows. Each part of the figure contains both the exact DNS subgrid variance together with the similarity predictions (25) employing the model constant (26). The curves marked (E) are averages of subgrid variances at the Eulerian grid points, while those marked (L) are averages of values interpolated to the Lagrangian droplet locations. The data in this figure correspond to simulation TP600 measured at time $t \Delta U_{0} / \delta_{\omega, 0}=85$ and the two filter widths are $\Delta_{f}=5 \Delta x$ and $\widehat{\Delta}_{f} / \Delta_{f}=2.2\left(C_{m}=0.5\right)$. Averaging the subgrid variances over planes tends to obfuscate some of the scatter that occurs in the actual local predictions; nevertheless, the results in Fig. 15 highlight several important attributes of the similarity model. First, the model yields good predictions for the magnitudes of all of the subgrid variances and at all planar locations within the mixing layer. Second, interpolating the model to the droplet locations yields equally accurate predictions compared to the Eulerian variance predictions; thus the model is valid for predicting fluctuation magnitudes felt by the droplets, a feature for which it has not previously been tested. Notice also that the model is able to capture the variance behavior both when the droplets show lesser variance than the Eulerian fields $\left(u_{i}\right.$ and $P$ ), as well as when the variances are larger at droplet locations $(\rho$, $T$, and $Y_{V}$ ). Lastly, the model is able to implicitly handle anisotropy of the gas phase velocity field since each $u_{i}$ components's variance is calculated from independent component similarity assumptions; this attribute would not be present if the subgrid velocity variances were computed from a transport equation for the subgrid kinetic energy, as only the summation of the three variances would then be known, as opposed to their individual magnitudes. Finally, Fig. 16 shows two sample model predictions of the same type as Fig. 15, but for simulation TP500b evaluated at the same time, but with $\widehat{\Delta_{f}} / \Delta_{f}=1.8\left(C_{m}=0.67\right)$. The results for these and all remaining (not shown) variable subgrid variances are equally accurate as those for simulation TP600. It therefore seems reasonable to use the scale similarity model to obtain the subgrid variances required to close the EIM extension.

\section{CONCLUSIONS}

Direct numerical simulations have been conducted of a temporally developing, droplet laden mixing layer at relatively large Reynolds numbers and mass loading ratios. Complete two-way couplings of mass, momentum, and energy are included in the formulation. As many as $18 \times 10^{6}$ grid points were used to discretize the Eulerian gas phase flow equations, and up to $5.7 \times 10^{6}$ individual evaporating droplets were tracked in the Lagrangian reference frame. These large resolutions could be achieved by utilizing a massively parallel architecture and they enabled the capture of the complete transition to mixing turbulence in a multiphase shear flow. Three-dimensional domain decomposition was used for the parallelization of the gas phase; the droplets were partitioned in sets according to the portion of the spatial domain they occupied, and therefore the equations of each set of thus defined droplets were solved by the processors 

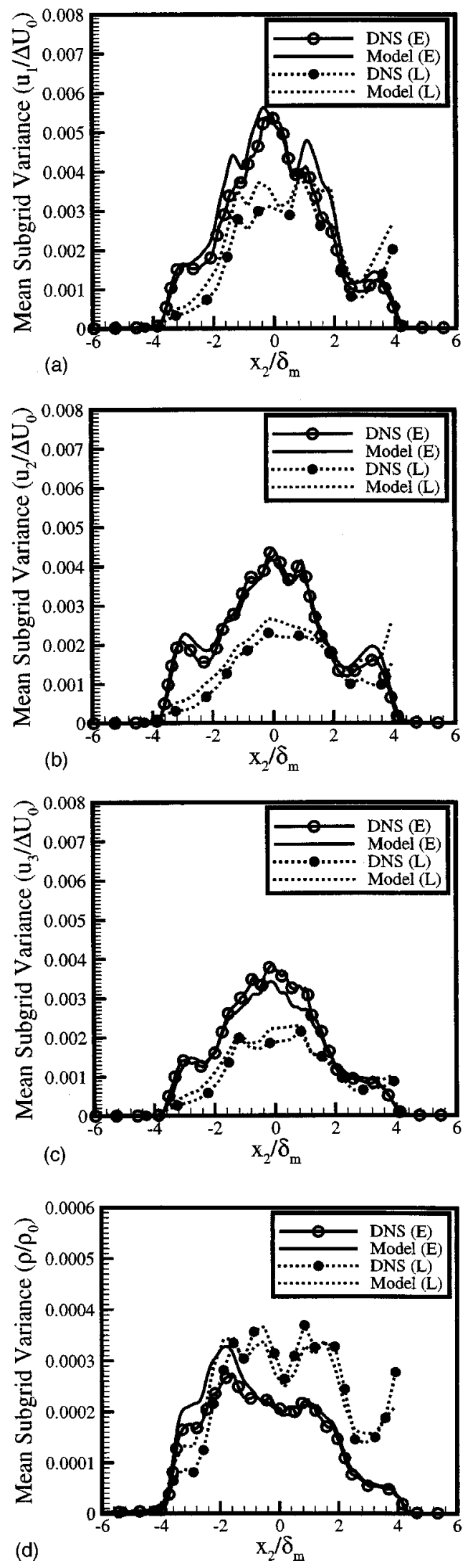
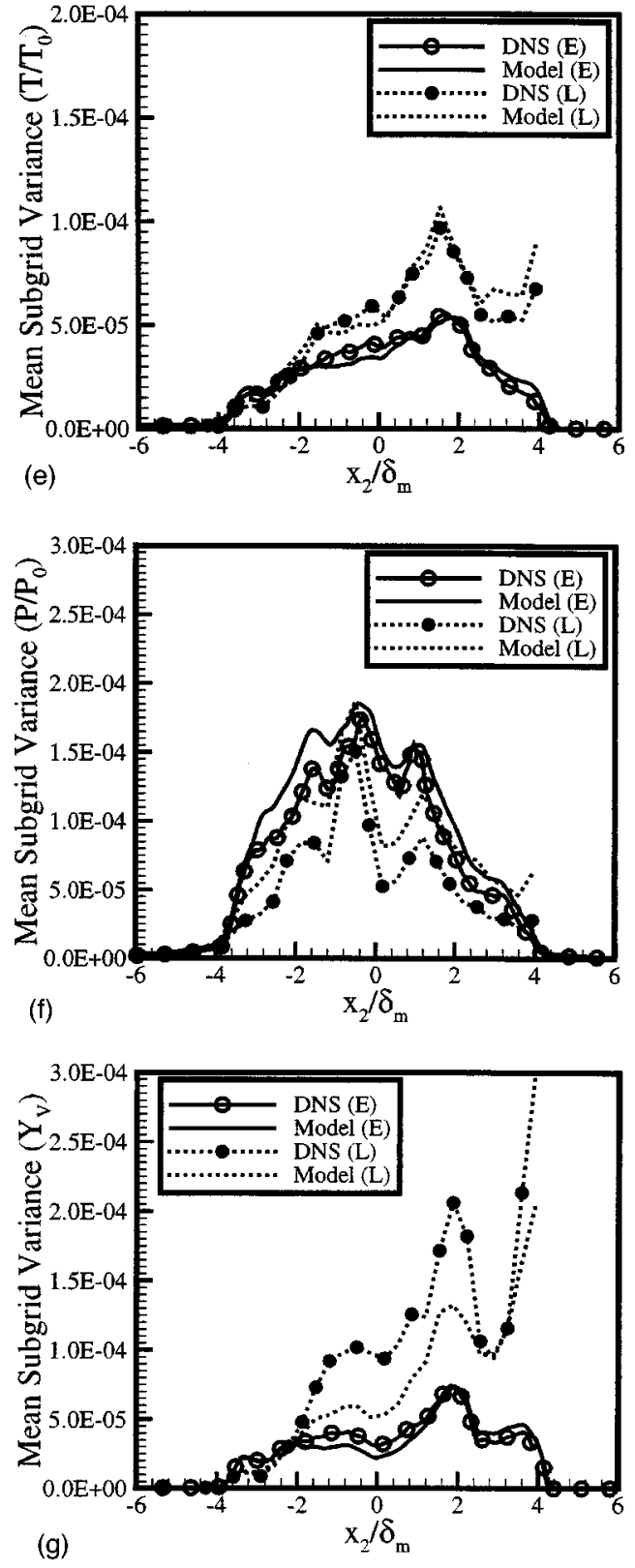

FIG. 15. A comparison of planar-averaged exact and modeled subgrid variances calculated at both Eulerian grid points and droplet locations for simulation TP600 at time $t \Delta U_{0} / \delta_{\omega, 0}=85$ with $\Delta_{f}=5 \Delta x \approx \delta_{\omega, 0} / 2$ and $\widehat{\Delta_{f}} / \Delta_{f}$ $=2.2$. (a) Streamwise velocity, (b) Cross stream velocity, (c) spanwise velocity, (d) density, (e) temperature, (f) pressure, and (g) vapor mass fraction. 

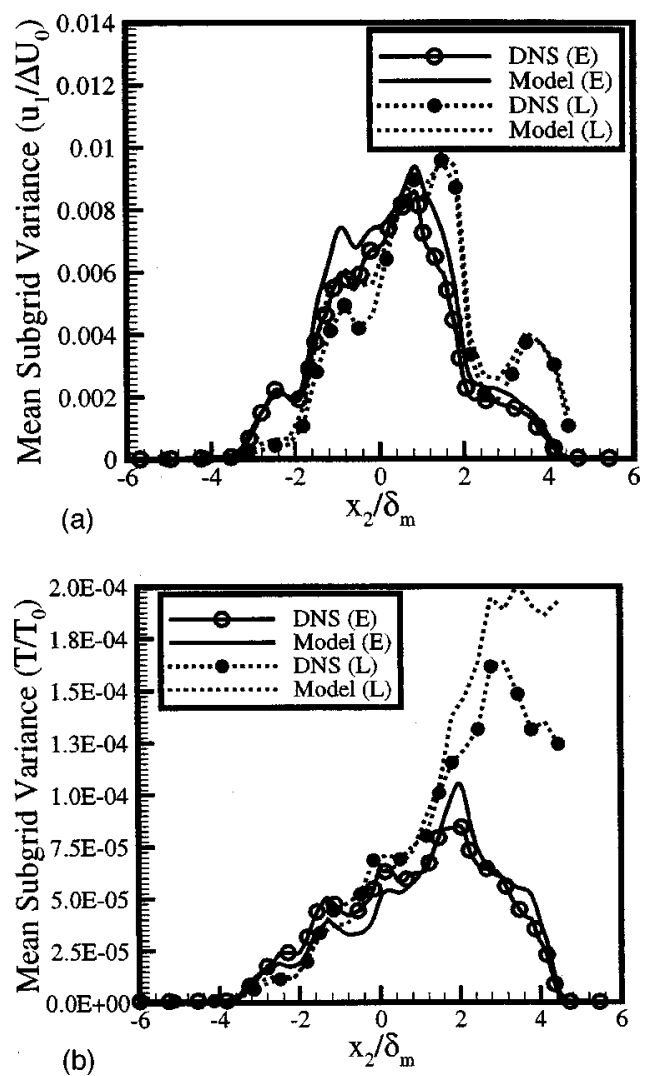

FIG. 16. A comparison of planar-averaged exact and modeled subgrid variances calculated at both Eulerian grid points and droplet locations for simulation TP500b at time $t \Delta U_{0} / \delta_{\omega, 0}=85$ with $\Delta_{f}=5 \Delta x \approx \delta_{\omega, 0} / 2$ and $\widehat{\Delta_{f}} / \Delta_{f}$ $=1.8$. (a) Streamwise velocity; (b) temperature.

associated with that domain. This procedure was shown to scale efficiently for both single- and two-phase simulations using as many as 64 CPUs.

The simulated flows were then used to study both the physics of mixing transition of droplet laden flow, and also to perform an a priori subgrid analysis pertinent to future large eddy simulations of two-phase flows. It was found that the mixing transition is characterized by a marked increase in the rotational energy of the flow that occurs between the times of the first primary spanwise rollup and the completion of the first pairing event. Transition of this type was observed for all but the lowest Reynolds number simulations. Increasing droplet mass loading ratios, at fixed Reynolds number, were shown to produce a more "natural" turbulence with lesser influences of the forcing perturbations on the long time flow fields. Furthermore, the rotational energy of the final time flows is also increased by the droplets due to both direct vorticity production and to additional disturbance wavelengths introduced by the coupling source terms. Finally, the relatively earlier saturation of large mass loading flows (at fixed $\mathrm{Re}_{0}$ ) results in a more organized preferential concentration of droplets within the mixing layer due to the larger inertia of these droplets.

The DNS database was then used to study the effects of subgrid Eulerian gas phase variables as they affect the individual droplet transport. It was shown that neglecting these effects may lead to significant errors in the droplet drag force, the droplet heating, and the evaporation rates in LES. This occurs even for Stokes numbers $\mathrm{St} \sim 1$ and relatively low gas temperatures; the conclusion is that these subgrid effects may require modeling in LES in order to accurately predict droplet/particle dispersion and vaporization. Therefore, such a subgrid model was constructed as an extension of the eddy interaction model to represent these effects in LES for both the gas velocity and thermodynamic subgrid fluctuations. In summary, the subgrid variance can be found by interpolating modeled variances from the Eulerian grid points to the local droplet locations and then employing a random number generator to produce "assumed" Gaussian distributed fluctuations for all of the variables. Each droplet will then interact with this fluctuation value for its "eddy residence time" obtained from its relative duration spent within the subgrid filter width, $\Delta_{f} /\left|\mathrm{v}_{i}\right|$. The assumption of Gaussian subgrid PDFs was tested with the DNS database and found to be adequate throughout most of the mixing layer, despite the fact that the ensemble of subgrid fluctuations are highly intermittent. The exception to this observation is the vapor mass fraction subgrid PDF, which shows substantial deviations from Gaussian behavior near the edges of the layer. Finally, the scale similarity model of Liu et al. ${ }^{33}$ was extended to predict the subgrid variances of all necessary variables. When based on a filter width-dependent model constant, the similarity model was shown to be reasonably accurate in capturing the variance behavior at both the Eulerian grid points and at the droplet locations based on interpolations.

\section{ACKNOWLEDGMENTS}

This research was conducted at the California Institute of Technology's Jet Propulsion Laboratory (JPL) and sponsored by General Electric (GE) through the Air Force Office of Scientific Research (AFOSR) Focused Research Initiative program with Dr. Hukam Mongia from GE serving as contract monitor. Computational resources were provided by the supercomputing facility at JPL.

${ }^{1}$ J. K. Eaton and J. R. Fessler, "Preferential concentration of particles by turbulence,"' Int. J. Multiphase Flow 20, 169 (1994).

${ }^{2}$ K. D. Squires and J. K. Eaton, "Particle response and turbulence modification in isotropic turbulence," Phys. Fluids A 2, 1191 (1990).

${ }^{3}$ S. Elghobashi and G. C. Truesdell, "'On the two-way interaction between homogeneous turbulence and dispersed solid particles. I: Turbulence modification," Phys. Fluids A 5, 1790 (1993).

${ }^{4}$ K. D. Squires and J. K. Eaton, "Effect of selective modification of turbulence on two-equation models for particle-laden turbulent flows," J. Fluids Eng. 116, 778 (1994).

${ }^{5}$ G. C. Truesdell and S. Elghobashi, "'On the two way interaction between homogeneous turbulence and dispersed solid particles. ii: Particle dispersion,"' Phys. Fluids 6, 1405 (1994).

${ }^{6}$ M. Boivin, O. Simonin, and K. D. Squires, "Direct numerical simulation of turbulence modulation by particles in isotropic turbulence,' J. Fluid Mech. 375, 235 (1998).

${ }^{7} \mathrm{~F}$. Mashayek, "Droplet-turbulence interactions in low Mach number homogeneous shear two-phase flows," J. Fluid Mech. 367, 163 (1998).

${ }^{8} \mathrm{~F}$. Mashayek, "Direct numerical simulations of evaporating droplet dispersion in forced low Mach number turbulence,' Int. J. Heat Mass Transf. 41, 2601 (1998).

${ }^{9}$ R. S. Miller, K. Harstad, and J. Bellan, "Evaluation of equilibrium and non-equilibrium evaporation models for many-droplet gas-liquid flow simulations,'” Int. J. Multiphase Flow 24, 1025 (1998). 
${ }^{10}$ R. S. Miller and J. Bellan, "Direct numerical simulation of a confined three-dimensional gas mixing layer with one evaporating hydrocarbondroplet laden stream," J. Fluid Mech. 384, 293 (1999).

${ }^{11}$ R. S. Miller and J. Bellan, "On the validity of the assumed pdf method for modeling binary mixing/reaction of evaporated vapor gas/liquid-droplet turbulent shear flow," Proceedings of the 27th Symposium (International) on Combustion, 1065-1072, 1998.

${ }^{12}$ W. Rodi, J. H. Ferziger, M. Breuer, and M. Pourquie, "Status of large eddy simulation: Results of a workshop," J. Fluids Eng. 119, 248 (1997).

${ }^{13} \mathrm{M}$. Lesieur and O. Metais, "New trends in large eddy simulations of turbulence,"' Annu. Rev. Fluid Mech. 28, 45 (1996).

${ }^{14} \mathrm{~F}$. Yeh and U. Lei, "On the motion of small particles in a homogeneous isotropic turbulent flow," Phys. Fluids A 3, 2571 (1991).

${ }^{15} \mathrm{~F}$. Yeh and U. Lei, "On the motion of small particles in a homogeneous turbulent shear flow," Phys. Fluids A 3, 2571 (1991).

${ }^{16}$ O. Simonin, E. Deutsch, and M. Boivin, "Large eddy simulation and second-moment closure model of particle fluctuating motion in two-phase turbulent shear flows," in Proc. 9th Symp. on Turbulent Shear Flows (Kyoto, Japan), 85, 1993.

${ }^{17}$ Q. Wang and K. D. Squires, "Large eddy simulation of particle-laden turbulent channel flow," Phys. Fluids 8, 1207 (1996).

${ }^{18}$ W. S. Uijttewaal and R. V. A. Oliemans, "Particle dispersion and deposition in direct numerical and large eddy simulations of vertical pipe flows," Phys. Fluids 8, 2590 (1996).

${ }^{19}$ Q. Wang and K. D. Squires, "Transport of heavy particles in a threedimensional mixing layer,' J. Fluids Eng. 120, 613 (1998).

${ }^{20} \mathrm{~S}$. Pannala and S. Menon, "Large eddy simulation of two-phase turbulent flows," AIAA Paper 98-0163, 1998.

${ }^{21}$ C. A. Kennedy and M. H. Carpenter, "Several new numerical methods for compressible shear-layer simulations," Appl. Numer. Math. 14, 397 (1994).

${ }^{22}$ V. G. McDonell, M. Adachi, and G. S. Samuelson, "Structure of reacting and nonreacting, nonswirling, air-assisted sprays, Part II: Drop behavior," Atomization Sprays 3, 411 (1993).

${ }^{23}$ R. D. Moser and M. M. Rogers, "Mixing transition and the cascade to small scales in a plane mixing layer," Phys. Fluids A 3, 1128 (1991).

${ }^{24}$ R. D. Moser and M. M. Rogers, "The three-dimensional evolution of a plane mixing layer: Pairing and transition to turbulence," J. Fluid Mech. 247, 275 (1993).

${ }^{25}$ M. M. Rogers and M. M. Moser, "Direct simulation of a self-similar turbulent mixing layer,'’ Phys. Fluids 6, 903 (1994).

${ }^{26}$ M. D. Slessor, C. L. Bond, and P. E. Dimotakis, "Turbulent shear-layer mixing at high Reynolds numbers: Effects of inflow conditions," J. Fluid Mech. 376, 115 (1998).

${ }^{27}$ K. D. Squires and J. K. Eaton, "Preferential concentration of particles by turbulence,' Phys. Fluids A 3, 1169 (1991).

${ }^{28}$ C. T. Crowe, J. N. Chung, and T. R. Troutt, "Particle dispersion by organized turbulent structures," in Particulate Two Phase Flow, edited by M. C. Roco, 1993, pp. 626-669.

${ }^{29}$ F. Wen, N. Kamalu, J. N. Chung, C. T. Crowe, and T. R. Troutt, "Particle dispersion by vortex structures in plane mixing layers,' J. Fluids Eng. 114, 657 (1992).

${ }^{30}$ B. Vreman, B. Geurts, and H. Kuerten, "A priori tests of large eddy simulation of the compressible plane mixing layer,' J. Eng. Math 29, 299 (1995).

${ }^{31}$ B. Vreman, B. Geurts, and H. Kuerten, "Large-eddy simulation of the turbulent mixing layer," J. Fluid Mech. 339, 357 (1997).

${ }^{32} \mathrm{~V}$. Borue and S. A. Orszag, "Local energy flux and subgrid-scale statistics in three-dimensional turbulence," J. Fluid Mech. 366, 1 (1998).

${ }^{33}$ S. Liu, C. Meneveau, and J. Katz, "On the properties of similarity subgrid-scale models as deduced from measurements in a turbulent jet," $\mathrm{J}$. Fluid Mech. 275, 83 (1994)

${ }^{34}$ J. O'Neil and C. Meneveau, "Subgrid-scale stresses and their modeling in a turbulent plane wake," J. Fluid Mech. 349, 253 (1997).

${ }^{35}$ J. Jimenez, A. Linan, M. M. Rogers, and F. J. Higuera, “A priori testing of subgrid models for chemically reacting non-premixed turbulent shear flows," J. Fluid Mech. 349, 149 (1997).

${ }^{36}$ D. L. Graham and P. W. James, "Turbulent dispersion of particles using eddy interaction models," Int. J. Multiphase Flow 22, 157 (1996).

${ }^{37}$ A. Berlemont, P. Desjonqueres, and G. Gouesbet, "Particle Lagrangian simulation in turbulent flows," Int. J. Multiphase Flow 16, 19 (1990).

${ }^{38}$ J. Jimenez, "Turbulent velocity fluctuations need not be Gaussian," J. Fluid Mech. 376, 139 (1998)

${ }^{39}$ Z. S. She, E. Jackson, and S. A. Orszag, "Structure and dynamics of homogeneous turbulence: Models and simulations," Proc. R. Soc. London, Ser. A 434, 101 (1991).

${ }^{40}$ R. S. Miller, S. H. Frankel, C. K. Madnia, and P. Givi, "JohnsonEdgeworth translation for probability modeling of binary mixing in turbulent flows," Combust. Sci. Technol. 91, 21 (1993).

${ }^{41}$ R. S. Miller, F. A. Jaberi, C. K. Madnia, and P. Givi, "The structure and the small-scale intermittency of passive scalars in homogeneous turbulence,' J. Sci. Comput. 10, 151 (1995)

${ }^{42}$ F. A. Jaberi, R. S. Miller, C. K. Madnia, and P. Givi, "Non-gaussian scalar statistics in homogeneous turbulence," J. Fluid Mech. 313, 241 (1996).

${ }^{43}$ C. K. Madnia and P. Givi, "'Direct numerical simulation and large eddy simulation of reacting homogeneous turbulence," in Large Eddy Simulation of Complex Engineering and Geophysical Flows, edited by B. Galperin and S. A. Orszag (Cambridge University Press, England, 1993), pp. 315-346.

${ }^{44}$ A. W. Cook and J. J. Riley, "A subgrid model for equilibrium chemistry in turbulent flows," Phys. Fluids 6, 2868 (1994).

${ }^{45}$ A. W. Cook and J. J. Riley, "Subgrid-scale modeling for turbulent reacting flows," Combust. Flame 112, 593 (1998).

${ }^{46}$ P. E. DesJardin and S. H. Frankel, "Large eddy simulation of a nonpremixed reacting jet: Application and assessment of subgrid-scale combustion models," Phys. Fluids 10, 2298 (1998).

${ }^{47}$ J. Bardina, J. H. Ferziger, and W. C. Reynolds, "Improved subgrid scale models for large eddy simulation," AIAA Paper 80-1357, 1980. 